\title{
Constraints on exact Lagrangians in cotangent bundles of manifolds fibered over the circle
}

Mihai Damian

\begin{abstract}
We give topological obstructions to the existence of a closed exact Lagrangian submanifold $L \hookrightarrow T^{*} M$, where $M$ is the total space of a fibration over the circle. For instance, we show that $\pi_{1}(L)$ cannot be the free product of two non-trivial groups and that the difference between the number of generators and the number of relations in a finite presentation of $\pi_{1}(L)$ is less than two.
\end{abstract}

Mathematics Subject Classification (2000). 57R17, 57R58, 57R70, 53D12.

Keywords. Lagrangian embeddings, Novikov homology, Floer homology.

\section{Introduction}

Let $M^{n}$ be a closed connected manifold and $T^{*} M$ its cotangent bundle endowed with the standard symplectic structure $\omega_{M}=d \lambda_{M}$, where $\lambda_{M}$ is the Liouville form $\lambda_{M}=\sum_{i} p_{i} d q_{i}$. Let $L^{n} \hookrightarrow T^{*} M$ be an exact Lagrangian submanifold, i.e. a submanifold such that $\lambda_{M} \mid L$ is an exact 1 -form.

The only known examples of exact Lagrangian submanifolds are the graphs of functions $f: M \rightarrow \mathbb{R}$

$$
L_{f}:=\left\{\left(q, d f_{q}\right) \mid q \in M\right\}
$$

and their images by Hamiltonian vector flows. The question of the existence of other examples was first evoked by V. I. Arnold in his survey "First steps in symplectic topology" [1]. It is far from being solved. A positive answer was given by R. Hind in the case $L=M=S^{2}$. The other related results which were proved up to now are topological obstructions to the existence of exact Lagrangian embeddings $L \hookrightarrow T^{*} M$. We summarize them in the statement below:

Theorem. Let $M$ be a closed manifold and $L \hookrightarrow T^{*} M$ an exact Lagrangian embedding of a closed manifold $L$. Denote by $p$ the projection of $L$ on the base space M. Then we have: 
(a) If $L$ and $M$ are orientable, then $\chi(L)=\operatorname{deg}^{2}(p) \chi(M)$. If $L$ and $M$ are not orientable the same equality is valid modulo 4.

(b) The index $\left[\pi_{1}(M): p_{*}\left(\pi_{1}(L)\right)\right]$ is finite.

(c) If $M$ is simply connected then $L$ can not be aspherical (i.e. Eilenberg-Mac Lane).

(d) If $M$ and $L$ are spin and $L$ has a vanishing Maslov class, then $H^{*}(L, K) \approx$ $H^{*}(M, K)$, for any field $K$ with $\operatorname{char}(K) \neq 2$.

(e) If $M$ is simply connected then $p^{*}: H^{2}(M) \rightarrow H^{2}(L)$ is injective and the index $\left[\pi_{2}(M): p_{*}\left(\pi_{2}(L)\right)\right]$ is finite.

The statement (a) was proved by M. Audin in [2], (b) was proved by F. Lalonde and J.-C. Sikorav in [12] and (c) is a result of C. Viterbo [24] (see also [23]). More recently, (d) was proved independently by K. Fukaya, P. Seidel and I. Smith [9], [10] and D. Nadler [15]. For $M=S^{n}$ and $L$ simply connected this was proved previously by P. Seidel [20] and by L. Buhovsky [3].

The assertion (e) was proved by A. Ritter [19]. The author uses some techniques coming from the Novikov homology theory which we will also do below.

The aim of this paper is to provide other obstructions in the case where $M$ is a total space of a fibration over the circle. Let us state our main results:

Theorem 1.1. Let $M^{n \geq 3}$ be a closed manifold which is the total space of a fibration over $S^{1}$ and let $L \hookrightarrow T^{*} M$ be an exact Lagrangian embedding of a closed manifold L. Then we have:

(a) Let $\left\langle g_{1}, g_{2}, \ldots, g_{p} \mid r_{1}, r_{2}, \ldots, r_{q}\right\rangle$ be an arbitrary presentation of the fundamental group $\pi_{1}(L)$. Then $p-q \leq 1$.

(b) The fundamental group $\pi_{1}(L)$ is not isomorphic to the free product $G_{1} * G_{2}$ of two non-trivial (finitely presented) groups.

Here are some examples of non-embedding statements which can be inferred from our result:

Corollary 1.2. Let $P, Q, L$ be closed manifolds and suppose that $\pi_{1}(P)$ is finite.

(a) Suppose that $\chi(L) \neq 0$. Then there is no exact Lagrangian embedding $L \times P \hookrightarrow$ $T^{*}\left(Q \times S^{1}\right)$.

In particular, let $\Sigma_{g}$ be a (not necessary orientable) surface of genus $g \geq 2$. Then there is no exact Lagrangian embedding of $\Sigma_{g} \times P$ into $T^{*}\left(Q \times S^{1}\right)$. More generally, for surfaces $\Sigma_{g_{i}}$ as above there is no exact Lagrangian embedding

$$
\Sigma_{g_{1}} \times \Sigma_{g_{2}} \times \cdots \times \Sigma_{g_{k}} \times P \hookrightarrow T^{*}\left(Q \times S^{1}\right) .
$$


(b) Let $L^{n \geq 4}$ be the connected sum $L_{1} \# L_{2}$ of two closed manifolds. Then there is no exact Lagrangian embedding $L \times P \hookrightarrow T^{*}\left(Q \times S^{1}\right)$ unless one of the $L_{i}$ is a simply connected $\mathbb{Z} / 2$-homology sphere.

(c) Suppose that there is an exact Lagrangian embedding

$$
L \times T^{l} \hookrightarrow T^{*}\left(T^{m} \times Q\right),
$$

where $T^{k}$ is the $k$-dimensional torus and $m>l$. Then L satisfies the properties (a) and (b) of Theorem 1.1.

1.1. Idea of the proof. Let $f: M \rightarrow S^{1}$ be a fibration. The closed 1-form $\alpha=f^{*} d \theta$ has no zeroes. Let $L$ be an exact embedding into $T^{*} M$. Consider the Lagrangian isotopy

$$
L_{t}=L+t \alpha .
$$

It follows that $L_{t} \cap L=\emptyset$ for $t$ large enough. The Lagrangian manifolds $L_{t}$ are not exact but they satisfy

$$
\left.\omega_{M}\right|_{\pi_{2}\left(T^{*} M, L_{t}\right)}=0
$$

just like an exact Lagrangian manifold. Under this hypothesis one can define a Floer-type complex $C_{\bullet}\left(L, L_{t}\right)$, which is spanned by the intersection points $L \cap L_{t}$. Therefore, this complex vanishes for $t \gg 0$.

It turns out that the homology of this complex is isomorphic to the Novikov homology $H_{*}\left(L, p^{*} u\right)$, where $u \in H^{1}(M, \mathbb{Z})$ is the cohomology class of $\alpha$ and $p: L \rightarrow M$ is the projection. In particular it is independent of $t$. It follows:

Theorem 1.3. $H_{*}\left(L, p^{*} u\right)=0$.

In order to prove Theorem 1.1 one has to argue in the following way: suppose that Theorem 1.1 (a) is false. Then one can show that the Novikov homology $H_{*}(L, v)$ does not vanish for any $v \in H^{1}(L, \mathbb{R})$, contradicting thus Theorem 1.3. A similar argument works for the proof of Theorem 1.1 (b).

The paper is organized as follows. In Section 2 we recall the definition and the main properties of Novikov homology. We prove the non-vanishing results needed in the above argument. In Section 3 we define the Floer complex $C_{\bullet}\left(L, L_{t}\right)$. Finally, in Section 4 we establish Theorem 1.3 and prove Theorem 1.1 and Corollary 1.2.

\section{Novikov theory}

2.1. Definition of Novikov homology. Let $u \in H^{1}(L ; \mathbb{R})$. Denote by $\Lambda$ the ring $\mathbb{Z} / 2\left[\pi_{1}(L)\right]$ and by $\hat{\Lambda}$ the ring of formal series $\mathbb{Z} / 2\left[\left[\pi_{1}(L)\right]\right]$. Consider a CW-decomposition of $L$ which we lift to the universal cover $\tilde{L}$. We get a $\Lambda$-free complex $C .(\tilde{L})$ spanned by (fixed lifts of) the cells of the triangulation of $L$. 
We define now the completed ring $\Lambda_{u}$ :

$$
\Lambda_{u}:=\left\{\lambda=\sum n_{i} g_{i} \in \hat{\Lambda} \mid g_{i} \in \pi_{1}(L), n_{i} \in \mathbb{Z} / 2, u\left(g_{i}\right) \rightarrow+\infty\right\} .
$$

The convergence to $+\infty$ means here that for all $A>0, u\left(g_{i}\right)<A$ only for a finite number of $g_{i}$ which appear with a non-zero coefficient in the sum $\lambda$.

Remark 2.1. Let $\lambda=1+\sum n_{i} g_{i} \in \Lambda_{u}$ where $u\left(g_{i}\right)>0$ for all $i$. Then $\lambda$ is invertible in $\Lambda_{u}$. Indeed, if we denote by $\lambda_{0}=\sum n_{i} g_{i}$ then it is easy to check that $\sum_{k \geq 0}\left(-\lambda_{0}\right)^{k}$ is an element of $\Lambda_{u}$ and it is obvious that it is the inverse of $\lambda$.

Definition. Let $C_{\bullet}(L, u)$ be the $\Lambda_{u}$-free complex $\Lambda_{u} \otimes_{\Lambda} C_{\bullet}(\tilde{L})$. The Novikov homology $H_{*}(L, u)$ is the homology of the complex $C_{\bullet}(L, u)$.

Remark 2.2. We may define in a similar way the Novikov homology with $\mathbb{Z}$ coefficients. As we want to compare it to Floer homology and the latter is defined for $\mathbb{Z} / 2$ coefficients we used $\mathbb{Z} / 2$ in the definitions above.

Now we prove:

Proposition 2.3. Let $L$ and $u$ be as above.

(a) Let $\left\langle g_{1}, g_{2}, \ldots, g_{p} \mid r_{1}, r_{2}, \ldots, r_{q}\right\rangle$ be a presentation of the fundamental group $\pi_{1}(L)$ which satisfies $p-q \geq 2$. Then, if $u \neq 0$ we have $H_{1}(L, u) \neq 0$.

(b) Suppose that $\pi_{1}(L)=G_{1} * G_{2}$, none of the $G_{i}$ being trivial. Then, if $u \neq 0$ we have $H_{1}(L, u) \neq 0$.

Proof. The presentation of $\pi_{1}(L)$ yields a CW decomposition of $L$ with one single zero-cell, $p$ one-cells and $q$ two-cells. Lifting it to the universal cover we see that the complex $C_{\bullet}(\tilde{L})$ ends like follows:

$$
\cdots \rightarrow \Lambda^{q} \stackrel{\delta_{2}}{\longrightarrow} \Lambda^{p} \stackrel{\delta_{1}}{\longrightarrow} \Lambda \rightarrow 0 .
$$

If $\left\{e_{i}\right\}_{i=1, \ldots, p}$ is the basis of $C_{1}=\Lambda^{p}$ given by the 1-cells and $\{e\}$ is the basis of $C_{0}=\Lambda$ given by the single 0 -cell, it is easy to see that the differential $\delta_{1}$ satisfies $\delta_{1}\left(e_{i}\right)=\left(1-g_{i}\right) e$.

(a) Since $u \neq 0, u\left(g_{i}\right) \neq 0$ for some $i$, we have that $1-g_{i}$ is invertible in $\Lambda_{u}$ (its inverse is $1+g_{i}+g_{i}^{2}+\cdots$ if $u\left(g_{i}\right)>0$, resp. $-g_{i}\left(1+g_{i}^{-1}+g_{i}^{-2}+\cdots\right)$ if $\left.u\left(g_{i}\right)<0\right)$. In particular in the tensored complex

$$
\cdots \rightarrow \Lambda_{u}^{q} \stackrel{\delta_{2}}{\longrightarrow} \Lambda_{u}^{p} \stackrel{\delta_{1}}{\longrightarrow} \Lambda_{u} \rightarrow 0 .
$$

the dimension of $\operatorname{Ker}\left(\delta_{1}\right)$ is $p-1$. Since $p-1>q$, it follows that $\operatorname{Im}\left(\delta_{2}\right) \neq \operatorname{Ker}\left(\delta_{1}\right)$, so $H_{1}(L, u) \neq 0$ as claimed. 
(b) Let $\left\{f_{j}\right\}_{j=1 \ldots, q}$ be the basis of $\Lambda^{q}$ defined by the two-cells corresponding to the relations $r_{1}, r_{2}, \ldots, r_{q}$. The matrix of $\delta_{2}$ with respect to $\left\{f_{j}\right\}$ and $\left\{e_{i}\right\}$ is given by the Fox derivatives $\partial r_{j} / \partial g_{i}$ [8]. These derivatives are defined by the following formulas:

$$
\begin{gathered}
\frac{\partial g_{i}}{\partial g_{i}}=1, \quad \frac{\partial g_{i}^{-1}}{\partial g_{i}}=-g_{i}^{-1}, \\
\frac{\partial\left(r r^{\prime}\right)}{\partial g_{i}}=\frac{\partial r}{\partial g_{i}}+r \frac{\partial r^{\prime}}{\partial g_{i}},
\end{gathered}
$$

for all $i=1, \ldots, p$,

where $r$ and $r^{\prime}$ are words in the letters $g_{i}^{ \pm 1}$.

Now suppose that $\pi_{1}(L)=G_{1} * G_{2}$ and consider (for $k=1,2$ ) finite presentations of $G_{k}$ with $p_{k}$ generators and $q_{k}$ relators. Denote by $\delta_{1}^{k}$ and $\delta_{2}^{k}$ the differentials of the complex (1) corresponding to these finite presentations. Then, for some $u: \pi_{1}(L) \rightarrow \mathbb{R}$, using the definition of the maps $\delta_{1}$ and $\delta_{2}$, we find that the complex (1) can be written as follows:

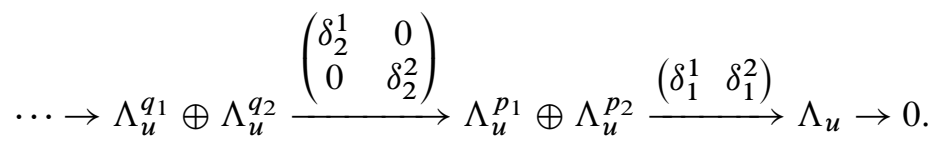

Suppose now that $u \neq 0$ and, without restricting the generality, that $\left.u\right|_{G_{1}} \neq 0$. As above the map $\delta_{1}^{1}$ is then surjective. This implies that for any $a \in \Lambda_{u}^{p_{2}}$ there is an element $b \in \Lambda_{u}^{p_{1}}$ such that $(b, a)$ belongs to the kernel of $\left(\begin{array}{ll}\delta_{1}^{1} & \delta_{1}^{2}\end{array}\right)$.

If $H_{1}(L, u)=0$, it follows that $\delta_{2}^{2}$ is an epimorphism. Now the sequence

$$
\Lambda_{u}^{q_{2}} \stackrel{\delta_{2}^{2}}{\longrightarrow} \Lambda_{u}^{p_{2}} \stackrel{\delta_{1}^{2}}{\longrightarrow} \Lambda_{u} \rightarrow 0
$$

is exact and therefore $\delta_{1}^{2}=0$. But this is impossible unless $G_{2}=0$ and the proof is finished.

2.2. Morse-Novikov theory. We recall in this subsection the relation between Novikov homology and closed 1-forms. Let $\alpha$ be a closed generic 1-form in the class $u \in H^{1}(L, \mathbb{R})$. "Generic" means here that the zeroes of $\alpha$ are of Morse type. Let $\xi$ be the gradient of $\alpha$ with respect to some generic metric on $L$. For every zero $c$ of $\alpha$ we fix a lift $\tilde{c}$ of $c$ in the universal cover $\tilde{L}$. We can define then a complex $C_{\bullet}(\alpha, \xi)$ spanned by the zeroes of $\alpha$ and graded by the Morse index: the "incidence number" $[d, c]$ for two zeroes of consecutive Morse indices is the (possibly infinite) sum $\sum n_{i} g_{i}$ where $n_{i}$ is the algebraic number of flow lines which join $c$ and $d$ and which are covered by a path in $\tilde{L}$ joining $g_{i} \tilde{c}$ and $\tilde{d}$. It turns out that this incidence number belongs to $\Lambda_{u}$, so $C_{\bullet}(\alpha, \xi)$ is actually a $\Lambda_{u}$-free complex.

The fundamental property of the Morse-Novikov theory was proved by S. P. Novikov in [16] and generalized by J.-C. Sikorav in [21]. The statement is: 
Theorem 2.4. For any generic couple $(\alpha, \xi)$ as above, the homology of the complex $C_{\bullet}(\alpha, \xi)$ is isomorphic to $H_{*}(L, u)$.

Remark 2.5. We may define in a similar way the Novikov homology $H_{*}(L, u)$ associated to a covering $\pi: \bar{L} \rightarrow L$ which satisfies $\pi^{*}(u)=0$ and we also may define $C_{\bullet}(\alpha, \xi)$ using the covering $\bar{L}$ instead of $\tilde{L}$. The statement of Theorem 2.4 holds in this more general setting.

An easy consequence of the above theorem is the following statement:

Proposition 2.6. Let $L_{1}, L_{2}$ be closed manifolds and let $u \in H^{1}\left(L_{1}, \mathbb{R}\right) \subset H^{1}\left(L_{1} \times\right.$ $\left.L_{2}, \mathbb{R}\right)$. Consider the Novikov homology $H_{*}\left(L_{1} \times L_{2}, u\right)$ associated to $u$ and to a covering $(\pi, I d): \bar{L}_{1} \times L_{2} \rightarrow L_{1} \times L_{2}$. Then

$$
H_{*}\left(L_{1} \times L_{2}, u\right) \approx H_{*}\left(L_{1}, u\right) \otimes_{\mathbb{Z} / 2} H_{*}\left(L_{2} ; \mathbb{Z} / 2\right) .
$$

In particular,

$$
H_{*}\left(L_{1} \times L_{2}, u\right)=0 \Longrightarrow H_{*}\left(L_{1}, u\right)=0 .
$$

Proof. Note first that the homologies $H_{*}\left(L_{1} \times L_{2}, u\right)$ and $H_{*}\left(L_{1}, u\right)$ are defined using the same Novikov ring $\Lambda_{u}$. Take a generic pair $\left(\alpha_{1}, \xi_{1}\right)$ associated to $u$ on $L_{1}$ and a generic pair $\left(d f_{2}, \xi_{2}\right)$ on $L_{2}$. One can easily see that the complex $C_{\bullet}\left(\alpha_{1}+d f_{2}, \xi_{1}+\xi_{2}\right)$ is isomorphic as a $\Lambda_{u}$-complex to the tensor product $C_{\bullet}\left(\alpha_{1}, \xi_{1}\right) \otimes_{\mathbb{Z} / 2} C_{\bullet}\left(d f_{2}, \xi_{2}\right)$. By comparing their homologies using the Künneth formula we get

$$
H_{*}\left(L_{1} \times L_{2}, u\right) \approx H_{*}\left(L_{1}, u\right) \otimes_{\mathbb{Z} / 2} H_{*}\left(L_{2} ; \mathbb{Z} / 2\right)
$$

as claimed

We end this section by the following trivial remark:

Remark 2.7. Let $L$ be a manifold with $\chi(L) \neq 0$. Then the Novikov homology, defined for any covering $\pi: \bar{L} \rightarrow L$, as above, does not vanish.

Indeed, the complex $C_{\bullet}(L, u)$ has the same Euler characteristic as $L$ and if the Novikov homology $H_{*}(L, u)$ vanishes, this Euler characteristic must be zero.

\section{The Floer complex}

In [14] Hong Van Le and Kaoru Ono defined a Floer complex spanned by the 1-periodic orbits of a symplectic non Hamiltonian vector field $X_{t}$ on a symplectic manifold $W$. They showed that, if $W$ is monotone, then this homology equals the Novikov 
homology associated to the cohomology class $\operatorname{Cal}\left(\phi_{t}\right)$; by $\operatorname{Cal}\left(\phi_{t}\right)$ we denote the Calabi invariant associated to the symplectic flow $\left(\phi_{t}\right)$ of $X_{t}$, defined by the formula

$$
\operatorname{Cal}\left(\phi_{t}\right)=\left[\int_{0}^{1} \beta_{t} d t\right],
$$

where $\beta_{t}$ is the symplectic dual of $X_{t}$ for $t \in[0,1]$. This is actually the image of the isotopy $\left(\phi_{t}\right)$ by the Flux morphism. By definition, the integral of $F \operatorname{lux}\left(\phi_{t}\right)$ over a loop $c: S^{1} \rightarrow W$ is the integral of the symplectic form over the cylinder $\phi_{t}(c)$.

Later, M. Pozniak [18] computed the (Lagrangian) Floer homology $H F\left(L_{1}, L_{2}\right)$ in the case where $L_{1}$ is the zero-section of $T^{*} M$ and $L_{2}=\phi\left(L_{1}\right), \phi$ being the time one map of a symplectic (non-Hamiltonian) isotopy.

Consider a closed exact Lagrangian manifold $L \hookrightarrow T^{*} M$ and denote by $L_{t}$ the image of $L$ through a symplectic isotopy $\left(\phi_{t}\right)$ on $T^{*} M$. Denote by $u \in H^{1}(M ; \mathbb{R})$ the class

$$
\left[\phi_{1}^{*} \lambda_{M}-\lambda_{M}\right] \in H^{1}\left(T^{*} M ; \mathbb{R}\right) \approx H^{1}(M ; \mathbb{R}) .
$$

It is not difficult to prove that this is actually the Calabi invariant $\operatorname{Cal}\left(\phi_{t}\right)$ for $W=$ $T^{*} M$. The goal of this section is the following

Theorem 3.1. Suppose that $L$ and $\phi_{1}(L)$ are transverse and that $u$ is rational (this means that the image of the morphism $u: \pi_{1}(M) \rightarrow \mathbb{R}$ is cyclic). Denote by $p^{*} u$ the composition $u \circ p: \pi_{1}(L) \rightarrow \mathbb{Z}$. There is a free $\Lambda_{p * u}$-complex $C_{\bullet}\left(L, \phi_{t}\right)$ spanned by the intersection points $L \cap \phi_{1}(L)$ and whose homology only depends on $L$ and on $u$.

Remark 3.2. The restriction $\operatorname{Im}(u) \approx \mathbb{Z}$ is not strong. Indeed, the isotopy $\left(\phi_{t}\right)$ is the flow of a vector field $X_{t}$ whose symplectic dual is a family of closed one forms $\beta_{t}$. If $\beta$ belongs to the class $u=\operatorname{Cal}\left(\phi_{t}\right)$, a well-known result of D. Tischler [22] asserts that there exists a closed 1 -form $\beta^{\prime}$, arbitrarily closed to $\beta$ and whose cohomology class $\left[\beta^{\prime}\right]$ is rational. This implies that given a symplectic isotopy $\left(\phi_{t}\right)$, there is an symplectic isotopy $\left(\phi_{t}^{\prime}\right)$ arbitrarily closed to it and with rational Calabi invariant: one can take $\left(\phi_{t}^{\prime}\right)$ defined by the family of closed one forms $\beta_{t}+\beta^{\prime}-\beta$.

Plan of the proof of Theorem 3.1. The complex $C_{\bullet}\left(L, \phi_{t}\right)$ will be the result of a version of Novikov homology theory on the (infinite dimensional) space of paths joining $L$ and $\phi_{1}(L)$. After some preliminary results proved in the next subsection we define in Section 3.2 an action 1-form $v$ whose zeroes are in one-to-one correspondence with the intersection points of $L$ and $\phi_{1}(L)$. In the following subsection we consider the gradient of $v$ with respect to a metric defined by a family of almost complex structures on $T^{*} M$. The "flow lines" of this gradient vector field will be actually holomorphic strips with boundary on $L \cup \phi_{1}(L)$. The results of Section 3.4 enable us to use this Floer-Novikov setting to define a free complex over the Novikov 
ring $\Lambda_{u}$ which is spanned by the points of $L \cap \phi_{1}(L)$ (Section 3.5). In Section 3.6 we prove that the homology of this complex only depends on $L$ and $u$. Finally, we show in Section 3.7 how the whole construction can be adapted in order to get a free complex over the Novikov ring $\Lambda_{p^{*} u}$, spanned by $L \cap \phi_{1}(L)$ and whose homology again only depends on $L$ and on $u$.

Let us now explain in detail how one defines the Floer complex $C_{\bullet}\left(L, \phi_{t}\right)$.

3.1. Preliminary results. Let $\left(\phi_{t}\right)$ be a symplectic isotopy as above and denote by $u \in H^{1}(M ; \mathbb{R})$ the class $\operatorname{Cal}\left(\phi_{t}\right)$ We prove the following lemma:

Lemma 3.3. There is a symplectic isotopy $\left(\psi_{t}\right)$ on $T^{*} M$ such that $\left.\psi_{1}\right|_{L}=\left.\phi_{1}\right|_{L}$ which is defined by (the symplectic dual of) $\beta=\alpha+d H_{t}$, where $\alpha \in u$ is a closed 1 -form on $M$ and $H: T^{*} M \times[0,1] \rightarrow \mathbb{R}$ has compact support.

Proof. The 1-form $\phi_{t}^{*} \lambda_{M}-\lambda_{M}$ is closed on $T^{*} M$. So, we may write

$$
\phi_{t}^{*} \lambda_{M}-\lambda_{M}=\alpha_{t}+d G_{t},
$$

where $G: T^{*} M \times[0,1] \rightarrow \mathbb{R}$ and the closed 1-form $\alpha_{t}$ is defined on $M$.

Define a symplectic isotopy $\Gamma_{t}: T^{*} M \rightarrow T^{*} M$ by

$$
\Gamma_{t}(p, q)=\left(p-\alpha_{t}(q), q\right) .
$$

Then, obviously, $\Gamma_{t}^{*} \lambda_{M}-\lambda_{M}=-\alpha_{t}$; Therefore, for $\chi_{t}=\Gamma_{t} \circ \phi_{t}$

$$
\chi_{t}^{*} \lambda_{M}=\phi_{t}^{*}\left(\lambda_{M}-\alpha_{t}\right)=\lambda_{M}+\alpha_{t}+d G_{t}-\phi_{t}^{*} \alpha_{t}=\lambda_{M}+d K_{t}
$$

for a smooth $K: T^{*} M \times[0,1] \rightarrow \mathbb{R}$, since $\phi_{t}^{*} \alpha_{t}$ and $\alpha_{t}$ are cohomologous.

Using the Lie derivative, one obtains easily that $\left(\chi_{t}\right)$ is a Hamiltonian isotopy. We want the isotopy $\left(\chi_{t}\right)$ (and in particular the function $K$ ) to be compactly supported. Since $L$ is compact, we may suppose that it is true and keep the relation $\chi_{t}=\Gamma_{t} \circ \phi_{t}$ valid on $L$. In other words, we have

$$
\chi_{t}=\Gamma_{t} \circ \tilde{\phi}_{t},
$$

where $\left.\tilde{\phi}_{t}\right|_{L}=\left.\phi_{t}\right|_{L}$ and $\chi_{t}$ is compactly supported.

On the other hand $\Gamma_{1}^{-1}(p, q)=\left(p+\alpha_{1}(q), q\right)$ is the time one of the symplectic isotopy $\widetilde{\Gamma}_{t}(p, q)=\left(p+t \alpha_{1}(q), q\right)$, so $\tilde{\phi}_{1}$ is the time one of

$$
\psi_{t}=\widetilde{\Gamma}_{t} \chi_{t}
$$

Outside a compact set $\psi_{t}(p, q)=\widetilde{\Gamma}_{t}(p, q)=\left(p+t \alpha_{1}(q), q\right)$ is defined by $\alpha_{1}$. It follows that $\left(\psi_{t}\right)$ is defined by $\alpha_{1}+d H_{t}$ for some smooth, compactly supported Hamiltonian $H: T^{*} M \times[0,1] \rightarrow \mathbb{R}$, and $\psi_{1}$ coincides with $\phi_{1}$, when restricted to $L$, as claimed. Moreover, $\left[\alpha_{1}\right]$ is obviously the class $\operatorname{Cal}\left(\phi_{t}\right)$ in $H^{1}(M ; \mathbb{R})$. The proof is finished. 
From now on, all the symplectic isotopies we consider have the property of Lemma 3.3 and rational Calabi invariant. In the following we consider the lift of such an isotopy to the universal cover of $T^{*} M$.

Remark 3.4. Let $j: L \hookrightarrow T^{*} M$ be a closed exact Lagrangian submanifold and let $p: L \rightarrow M$ be the projection on the base space. Without restricting the generality, we may add the hypothesis $\pi_{1}\left(T^{*} M, L\right)=0$ in the statement of Theorem 1.1. Indeed, by (b) of Theorem, we know that the index $\left[\pi_{1}(M): p\left(\pi_{1}(L)\right)\right]$ is finite. Consider the finite cover $\bar{M} \rightarrow M$ which corresponds to the subgroup $p\left(\pi_{1}(L)\right)$. Then, it is easy to show that there is an exact Lagrangian embedding $L \hookrightarrow T^{*} \bar{M}$ which is a lifting of $L \hookrightarrow T^{*} M$ (the definition of this lifting is similar to the one in 2.2 of [12]). This embedding induces an epimorphism $\pi_{1}(L) \rightarrow \pi_{1}(\bar{M})$. Since $\bar{M}$ is still a total space of a fibration over the circle, we may prove Theorem 1.1 for $\bar{M}$ instead of $M$ in order to get the desired obstructions on $L$.

Consider now the universal cover $\pi: \tilde{M} \rightarrow M$ and the induced projection $\tilde{\pi}: T^{*} \tilde{M} \rightarrow T^{*} M$. Denote by $y \mapsto g y$ the diffeomorphism of $T^{*} \tilde{M}$ defined by the action of $g$ on $\pi_{1}\left(T^{*} M\right) \approx \pi_{1}(M)$. Since this is a right action, one should keep in mind that $g^{\prime}\left(g^{\prime \prime} y\right)=\left(g^{\prime \prime} g^{\prime}\right) y$. Let $K$ be the kernel of the epimorphism $p: \pi_{1}(L) \rightarrow \pi_{1}(M)$ and let $\bar{\pi}: \bar{L} \rightarrow L$ be the cover of $L$ associated to $K$. According to Remark 3.4 this is a $\pi_{1}(M)$-covering, so $\bar{L}$ is not compact. We prove

Lemma 3.5. There exists an exact Lagrangian embedding $\Psi: \bar{L} \hookrightarrow T^{*} \tilde{M}$ which is a lifting of $L \hookrightarrow T^{*} M$. Moreover, we have $\Psi(\bar{L})=\tilde{\pi}^{-1}(j(L))$ and for any $g \in \pi_{1}(M), \bar{x} \in \bar{L}$,

$$
\Psi(g \bar{x})=g \cdot \Psi(\bar{x}) .
$$

Proof. Consider the pullback of $\tilde{\pi}: T^{*} \tilde{M} \rightarrow T^{*} M$ by the embedding $j: L \rightarrow$ $T^{*} M$ i.e. the restriction to $L$ of the covering $T^{*} \tilde{M} \rightarrow T^{*} M$. Since $p: \pi_{1}(L) \rightarrow$ $\pi_{1}(M)$ is an epimorphism, this space is connected. It follows that this covering of $L$ is isomorphic to $\bar{L} \rightarrow L$. We keep the same notation $\bar{L}$ and consider the canonical maps $\Psi: \bar{L} \rightarrow T^{*} \tilde{M}$ and $\bar{\pi}: \bar{L} \rightarrow L$. Note that, for $g \in \pi_{1}(M)$ we have

$$
\Psi(g \bar{x})=g \cdot \Psi(\bar{x}),
$$

as claimed. Using the commutative diagram

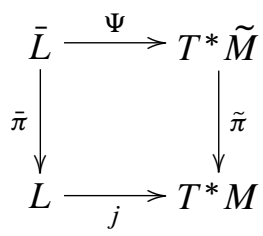


one easily checks that $\Psi$ is an exact Lagrangian embedding. The equality $\Psi(\bar{L})=$ $\tilde{\pi}^{-1}(j(L))$ is an obvious consequence of the definition of the pullback.

Notations. We use the same notation $\bar{L} \subset T^{*} \tilde{M}$ for the Lagrangian submanifold which is the image $\Psi(\bar{L})$ of the embedding constructed above. To keep the notations uniform, we will denote by $\bar{a}$ a lift to $T^{*} \tilde{M}$ of an object $a \in T^{*} M$.

Consider now a symplectic isotopy $\left(\phi_{t}\right)$ on $T^{*} M$ which is defined by $\alpha+d H_{t}$, as in Lemma 3.3. The following result is straightforward:

Lemma 3.6. The symplectic isotopy $\left(\phi_{t}\right)$ lifts to a Hamiltonian isotopy $\left(\bar{\phi}_{t}\right)$ on $T^{*} \tilde{M}$. Moreover, if we denote $L_{t}=\phi_{t}(L)$ and $\bar{L}_{t}=\bar{\phi}_{t}(\bar{L})$, then

$$
\bar{L} \cap \bar{L}_{1}=\bigcup_{x \in L \cap L_{1}} \tilde{\pi}^{-1}(x) .
$$

Proof. Let $\alpha+d H_{t}$ be the closed 1-form on $T^{*} M$ whose symplectic dual $X_{t}$ defines $\left(\phi_{t}\right)$. Take its pullback $\tilde{\pi}^{*} \alpha+d\left(H_{t} \circ \tilde{\pi}\right)$ on $T^{*} \tilde{M}$. The flow of its symplectic dual $\bar{X}_{t}$ defines a symplectic isotopy $\tilde{\phi}_{t}$ which is actually Hamiltonian since $T^{*} \tilde{M}$ is simply connected. It is obvious that $\tilde{\pi}_{*}\left(\bar{X}_{t}\right)=X_{t}$, which immediately implies that $\left(\bar{\phi}_{t}\right)$ is a lift of $\left(\phi_{t}\right)$. We have thus a commutative diagram

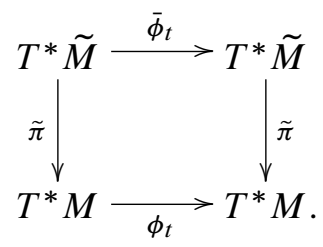

Now, following the proof of Lemma 3.5, we have $\bar{L}=\tilde{\pi}^{-1}(L)$, and using the above diagram

$$
\bar{L}_{t}=\bar{\phi}_{t}(\bar{L})=\bar{\phi}_{t}\left(\tilde{\pi}^{-1}(L)\right)=\tilde{\pi}^{-1}\left(\phi_{t}(L)\right)=\tilde{\pi}^{-1}\left(L_{t}\right) .
$$

It follows that

$$
\bar{L} \cap \bar{L}_{1}=\tilde{\pi}^{-1}\left(L \cap L_{1}\right),
$$

as claimed.

3.2. The action 1-form. Let $L \hookrightarrow T^{*} M$ be closed exact Lagrangian and let $\left(\phi_{t}\right)$ be a symplectic isotopy as above. Denote by $u \in H^{1}(M ; \mathbb{R})$ the class $\operatorname{Cal}\left(\phi_{t}\right)$. We suppose that $\pi_{1}\left(T^{*} M, L\right)=0$, using Remark 3.4.

For $L_{t}=\phi_{t}(L)$, denote

$$
\Omega\left(L_{0}, L_{1}\right)=\left\{z \in \mathcal{C}^{\infty}\left([0,1], T^{*} M\right) \mid z(i) \in L_{i}, i=0,1\right\} .
$$


Define a 1-form on $\Omega\left(L_{0}, L_{1}\right)$ by

$$
v_{z}(V)=\int_{0}^{1} \omega_{M}\left(z^{\prime}(t), V(t)\right) d t .
$$

The zeroes of $v$ are the constant paths corresponding to the intersection points $L_{0} \cap L_{1}$.

Let $\gamma: S^{1} \rightarrow \Omega\left(L_{0}, L_{1}\right)$ be a loop. We see this loop as a map $\gamma: S^{1} \times[0,1] \rightarrow$ $T^{*} M$. We prove

Lemma 3.7. We have

$$
\int_{\gamma} v=-u\left(\gamma\left(S^{1} \times\{0\}\right)\right)
$$

In particular $v$ is closed.

Proof. Let us evaluate $\int_{\gamma} \nu$. We denote by $(s, t)$ the coordinates on $S^{1} \times[0,1]$.

$$
\int_{\gamma} v=\int_{0}^{1} v(\partial \gamma / \partial s) d s=\int_{0}^{1} \int_{0}^{1} \omega(\partial \gamma / \partial t, \partial \gamma / \partial s) d t d s=-\int_{\gamma\left(S^{1} \times[0,1]\right)} \omega_{M} .
$$

Using Stokes' theorem we find that

$$
\int_{\gamma} v=-\int_{\gamma\left(S^{1} \times\{1\}\right)} \lambda_{M}+\int_{\gamma\left(S^{1} \times\{0\}\right)} \lambda_{M} .
$$

Since $L=L_{0}$ is exact, the second integral in the right term above vanishes. The first one can be written as follows:

$$
\begin{aligned}
\int_{\gamma\left(S^{1} \times\{1\}\right)} \lambda_{M} & =\int_{\phi_{1}^{-1}\left(\gamma\left(S^{1} \times\{1\}\right)\right)} \phi_{1}^{*} \lambda_{M} \\
& =\int_{\phi_{1}^{-1}\left(\gamma\left(S^{1} \times\{1\}\right)\right)} \lambda_{M}+\int_{\phi_{1}^{-1}\left(\gamma\left(S^{1} \times\{1\}\right)\right)}\left(\phi_{1}^{*} \lambda_{M}-\lambda_{M}\right) .
\end{aligned}
$$

As above, the first integral in the right term is zero. Since $\phi_{1}^{*} \lambda_{M}-\lambda_{M}$ is a closed 1 -form belonging to the cohomology class $u=\operatorname{Cal}\left(\phi_{t}\right)$ the second integral equals $u\left(\phi_{1}^{-1}\left(\gamma\left(S^{1} \times\{1\}\right)\right)=u\left(\gamma\left(S^{1} \times\{0\}\right)\right)\right.$. Finally

$$
\int_{\gamma} v=-u\left(\gamma\left(S^{1} \times\{0\}\right)\right)
$$

and the proof of the lemma is finished.

Now let $\left(\bar{L}_{t}\right)$ be the lifting of $\left(L_{t}\right)$ to $T^{*} \tilde{M}$, like in the preceding section and define $\Omega\left(\bar{L}_{0}, \bar{L}_{1}\right)$ as above. Also define the 1 -form $\bar{v}$ on $\Omega\left(\bar{L}_{0}, \bar{L}_{1}\right)$ in a similar way. The zeroes of $\bar{v}$ are therefore in bijection with the intersection points $\bar{L}_{0} \cap \bar{L}_{1}$. 
For the canonical projection $\pi^{\Omega}: \Omega\left(\bar{L}_{0}, \bar{L}_{1}\right) \rightarrow \Omega\left(L_{0}, L_{1}\right)$ we obviously have

$$
\left(\pi^{\Omega}\right)^{*} v=\bar{v} .
$$

Also remark that $\bar{v}$ is exact by Lemma 3.7. Denote by $\mathcal{A}$ a primitive of $\bar{v}$.

There is an action of $\pi_{1}(M)$ on $\Omega\left(\bar{L}_{0}, \bar{L}_{1}\right)$, coming from the action of $\pi_{1}(M)$ on $T^{*} \tilde{M}$. We show:

Lemma 3.8. Let $z \in \Omega\left(\bar{L}_{0}, \bar{L}_{1}\right)$ and $g \in \pi_{1}(M)$. Then

$$
\mathcal{A}(g \cdot z)=\mathcal{A}(z)-u(g) .
$$

Proof. Let $\bar{\gamma}$ be a path between $z$ and $g \cdot z$ in $\Omega\left(\bar{L}_{0}, \bar{L}_{1}\right)$ (which does exist since $T^{*} \tilde{M}$ is simply connected). Denote $\gamma=\pi^{\Omega}(\bar{\gamma})$. We have

$$
\mathcal{A}(g \cdot z)-\mathcal{A}(z)=\int_{\bar{\gamma}} \bar{v}=\int_{\gamma} v .
$$

Now $\gamma$ is a loop in $\Omega\left(L_{0}, L_{1}\right)$ which has the property that $\gamma\left(S^{1} \times\{t\}\right)$ represents $g \in \pi_{1}(M)$ for every $t \in[0,1]$. By applying Lemma 3.7, we get the desired relation.

Alternatively, one may consider another action 1-form. If the isotopy $\left(\phi_{t}\right)$ is defined by the symplectic dual $X_{t}$ of $\alpha+d H_{t}$ (defined by $\left.\omega_{M}\left(\cdot, X_{t}\right)=\left(\alpha+d H_{t}\right)(\cdot)\right)$, we define a 1-form on $\Omega(L, L)$ by:

$$
\hat{v}_{z}(V)=\int_{0}^{1} \omega_{M}\left(z^{\prime}(t), V(t)\right)+\left(\alpha+d H_{t}\right)(V(t)) d t .
$$

The zeroes of $\hat{v}$ are the flow trajectories starting from $L$ and ending in $L$ at time $t=1$, which means that there are in bijection with $L_{0} \cap L_{1}$.

As in Lemma 3.7, for a loop $\gamma$ in $\Omega(L, L)$ we have

$$
\int_{\gamma} \hat{v}=-\int_{S^{1} \times[0,1]} \gamma^{*} \omega_{M}+\int_{S^{1}} \int_{0}^{1}\left(\alpha+d H_{t}\right)(\partial \gamma / \partial s) d t d s
$$

The first integral in the right term is zero, as in the proof of Lemma 3.7. The second one equals

$$
\int_{0}^{1} \int_{S^{1}} \alpha(\partial \gamma / \partial s) d s d t=\int_{0}^{1} \int_{\gamma(\cdot, t)} \alpha d t=\int_{\gamma(\cdot, 0)} \alpha=u(\gamma(\cdot, 0)),
$$

since $\int_{\gamma(\cdot, t)} \alpha$ does not depend on $t$. 
This relation implies that $\hat{v}$ is closed. Then, as above, one defines a 1-form on $\Omega(\bar{L}, \bar{L})$ in the similar way and obtains that $\pi_{\Omega}^{*} \hat{v}=d \hat{\mathcal{A}}$. As in Lemma 3.8 we infer that any primitive $\hat{\mathcal{A}}$ satisfies the equality

$$
\hat{\mathcal{A}}(g \cdot z)=\hat{A}(z)+u(g) .
$$

These two approaches are strongly related, as it can be seen from the following remark.

Remark 3.9. Let $v_{-}$be the 1 -form defined in the same way as $v$ on $\Omega\left(L_{0}, \phi_{1}^{-1}\left(L_{0}\right)\right)$. The map $\Gamma(z)=\phi_{t}^{-1} z$ is obviously a bijection between $\Omega(L, L)$ and $\Omega\left(L_{0}, \phi_{1}^{-1}\left(L_{0}\right)\right)$. We have the relation

$$
\Gamma^{*} \nu_{-}=\hat{v} .
$$

The fact that this relation is given by a bijection which is defined using the form $v_{-}$ and the isotopy $\left(\phi_{t}^{-1}\right)$ (whose Calabi invariant is $-u$ ) explains the change of sign in the analogue of Lemma 3.8 above. The proof is straightforward.

3.3. The gradient. Let $\left(J_{t}\right)_{t \in[0,1]}$ be a family of almost complex structures on $T^{*} M$ which are compatible with $\omega_{M}$. This means that $g_{t}(X, Y)=\omega_{M}\left(X, J_{t} Y\right)$ are Riemannian metrics on $T^{*} M$. Define then a metric $g^{\Omega}$ on $\Omega\left(L_{0}, L_{1}\right)$ by

$$
g^{\Omega}(V, W)=\int_{0}^{1} g_{t}(V(t), W(t)) d t .
$$

The gradient of $v$ with respect to this metric is given by

$$
\operatorname{grad}_{z}^{g^{\Omega}} v=J_{t}(z) z^{\prime} .
$$

The trajectories of the time dependent vector field $X_{t}=-\operatorname{grad}_{z}^{g \Omega} v$ on $\Omega\left(L_{0}, L_{1}\right)$ can be seen as maps $v$ of two variables $(s, t)$ satisfying the Cauchy-Riemann equation. More precisely $v$ is a solution of

$$
\left\{\begin{array}{l}
\frac{\partial v}{\partial s}+J_{t}(v) \frac{\partial v}{\partial t}=0 \\
v(s, 0) \in L_{0} \\
v(s, 1) \in L_{1}
\end{array}\right.
$$

For $v: \mathbb{R} \times[0,1] \rightarrow T^{*} M$, solution of $(*)$ one defines the energy $E(v)$ by the formula

$$
E(v)=\int_{\mathbb{R} \times[0,1]}\left\|\frac{\partial v}{\partial s}\right\|_{g_{t}}^{2} d s d t .
$$

One can easily see that

$$
E(v)=\int_{\mathbb{R} \times[0,1]}\left\|\frac{\partial v}{\partial t}\right\|_{g_{t}}^{2} d s d t=\int_{\mathbb{R} \times[0,1]} v^{*} \omega_{M} .
$$


Denote by $\mathcal{M}$ the space of solutions of finite energy:

$$
\mathcal{M}\left(L_{0}, L_{1}\right)=\left\{v \in \mathcal{C}^{\infty}\left(\mathbb{R} \times[0,1], T^{*} M\right) \mid v \text { satisfies }(*) ; E(v)<+\infty\right\}
$$

For $x, y \in L_{0} \cap L_{1}$ define

$\mathcal{M}(x, y)=\left\{v \in \mathcal{C}^{\infty}\left(\mathbb{R} \times[0,1], T^{*} M\right) \mid v\right.$ satisfies $\left.(*) ; \quad \begin{array}{l}\lim _{s \rightarrow-\infty} v(s, \cdot)=x \\ \lim _{s \rightarrow+\infty} v(s, \cdot)=y\end{array}\right\}$.

As in [4] (Proposition 1.b), [17] (Proposition 3.2), we have

\section{Theorem 3.10.}

$$
\mathcal{M}\left(L_{0}, L_{1}\right)=\bigcup_{x, y \in L_{0} \cap L_{1}} \mathcal{M}(x, y) .
$$

Note that the solutions of $\mathcal{M}\left(L_{0}, L_{1}\right)$ with vanishing energy are exactly the critical points of $\mathscr{A}_{H}$ i.e. the constant paths given by the intersection points $L_{0} \cap L_{1}$. However, for $x \in \operatorname{Crit}\left(\mathcal{A}_{H}\right)$ the space $\mathcal{M}(x, x)$ may also contain solutions of non zero energy. It is useful to consider the spaces of solutions with non vanishing energy:

$$
\mathcal{M}^{*}(x, y)= \begin{cases}\mathcal{M}(x, y) & \text { for } x \neq y, \\ \mathcal{M}(x, x) \backslash\{x\} & \text { for } x=y .\end{cases}
$$

The family $\left(J_{t}\right)_{t \in[0,1]}$ can be lifted to a family of compatible almost complex structures $\left(\bar{J}_{t}\right)_{t \in[0,1]}$ on $T^{*} \tilde{M}$. We define then the spaces of solutions $\mathcal{M}\left(\bar{L}_{0}, \bar{L}_{1}\right)$ and $\mathcal{M}^{*}(\bar{x}, \bar{y})$ as above. Note that $\mathcal{M}(\bar{x}, \bar{x})=\{\bar{x}\}$ (see the formula for the energy below). The analogue of Theorem 3.10 remains valid.

Obviously, the projection $\tilde{\pi}$ maps $\mathcal{M}\left(\bar{L}_{0}, \bar{L}_{1}\right)$ to $\mathcal{M}\left(L_{0}, L_{1}\right)$ and if $\tilde{\pi}(\bar{v})=v$ then $E(\bar{v})=E(v)$. Moreover, one can easily see that any solution $v \in \mathcal{M}\left(L_{0}, L_{1}\right)$ lifts to a solution $\bar{v} \in \mathcal{M}\left(\bar{L}_{0}, \bar{L}_{1}\right)$. Also remark that, if $\bar{v} \in \mathcal{M}(\bar{x}, \bar{y})$, then we have

$$
E(v)=-\int_{-\infty}^{+\infty} \frac{\partial}{\partial s} \mathcal{A}(\bar{v}(s, \cdot)) d s=\mathcal{A}(\bar{x})-\mathcal{A}(\bar{y}) .
$$

Alternatively, we can consider the gradient of the 1 -form $\hat{v}$ with respect to a family of metrics $\hat{g}^{\Omega}$, defined by a family of compatible almost complex structures $\left(\hat{J}_{t}\right)$, as above. This approach leads to the perturbed Cauchy-Riemann equation:

$$
\left\{\begin{array}{l}
\frac{\partial \hat{v}}{\partial s}+J_{t}(\hat{v})\left(\frac{\partial \hat{v}}{\partial t}-X_{t}(\hat{v})\right)=0 \\
\hat{v}(s, 0) \in L \\
\hat{v}(s, 1) \in L
\end{array}\right.
$$

We define the energy of the solution $\hat{v}$ of $(* *)$ by the same formula and then analogously the spaces of solutions $\widehat{\mathcal{M}}(L, L)$ and $\widehat{\mathcal{M}}(x, y)$. The statement of Theorem 3.10 remains true in this setting. 
Again, for a solution $\bar{v}$ of $(* *)$ joining $\bar{x}, \bar{y} \in \Omega(\bar{L}, \bar{L})$ upstairs, we have

$$
E(\bar{v})=\hat{\mathcal{A}}(\bar{x})-\hat{\mathcal{A}}(\bar{y})
$$

and these solutions are liftings of the solutions of $(* *)$ on $T^{*} M$.

Remark 3.11. Let $\hat{v} \in \widehat{\mathcal{M}}(L, L)$. Define $v(s, t)=\phi_{t}^{-1}(\hat{v}(s, t))$. Consider two families of compatible almost complex structures $\left(J_{t}\right)$ and $\left(\hat{J}_{t}\right)$ on $T^{*} M$ which are related by

$$
\hat{J}_{t}(\cdot)=\left(\phi_{t}\right)_{*} J_{t}\left(\phi_{t}^{-1}\right)_{*}(\cdot) \text {. }
$$

Then one easily checks the equality

$$
\frac{\partial v}{\partial s}+J_{t} \frac{\partial v}{\partial t}=\left(\phi_{t}^{-1}\right)_{*}\left[\frac{\partial \hat{v}}{\partial s}+\hat{J}_{t}\left(\frac{\partial \hat{v}}{\partial t}-X_{t}(\hat{v})\right)\right],
$$

and, furthermore, the equality

$$
E(\hat{v})=E(v)
$$

We infer that the mapping $\hat{v} \mapsto v$ is a one-to-one correspondence between $\widehat{\mathcal{M}}(L, L)$ and $\mathcal{M}\left(L_{0}, \phi_{1}^{-1}\left(L_{0}\right)\right)$ for the given choices of the almost complex families.

One can see this bijection as a direct consequence of the relation $\Gamma^{*} \nu_{-}=\hat{v}$ from Remark 3.9 for appropriate choices of metrics on the paths spaces $\Omega(\cdot, \cdot)$.

3.4. Transversality and compactness. Suppose that the manifolds $L_{0}$ and $L_{1}$ are transverse. Then we can prove the following theorem in the same manner as in [6] (see also [17]).

Theorem 3.12. Under the transversality assumption above, and for a generic choice of $J_{t}$ the spaces $\mathcal{M}^{*}(x, y)$ are finite dimensional manifolds of local dimension $\mu(v)=$ the Maslov-Viterbo index of $v$ (see [25] for the definition). The same is true for $\mathcal{M}(\bar{x}, \bar{y})$ (note that $\mathcal{M}(\bar{x}, \bar{x})=\{\bar{x}\})$. The map $\tilde{\pi}$ induces an embedding

$$
\tilde{\pi}: \mathcal{M}(\bar{x}, \bar{y}) \rightarrow \mathcal{M}(x, y),
$$

for $\tilde{\pi}(\bar{x})=x$ and $\tilde{\pi}(\bar{y})=y$.

Remark 3.13. Using Remark 3.11 we infer from the preceding theorem that the spaces $\widehat{\mathcal{M}}^{*}(x, y)$ are manifolds for a generic choice of $\hat{J}_{t}$.

The map $\sigma \mapsto v(\sigma+\cdot, \cdot)$ defines an action of $\mathbb{R}$ on $\mathcal{M}(x, y)$. Denote by $\mathscr{L}(x, y)$ the quotient $\mathcal{M}^{*}(x, y) / \mathbb{R}$. Analogously, let $\overline{\mathscr{L}}(\bar{x}, \bar{y})$ be the quotient of $\mathcal{M}^{*}(\bar{x}, \bar{y})$. As the action of $\mathbb{R}$ is free on $\mathcal{M}^{*}(x, y)$, we infer from Theorem 3.12 that $\mathscr{L}(x, y)$ and $\overline{\mathscr{L}}(\bar{x}, \bar{y})$ are finite dimensional manifolds. 
In order to define the differential of $C_{\bullet}\left(L_{0}, L_{1}\right)$, we need to study the compactness of the trajectory spaces $\mathscr{L}(x, y)$. Remark that, since $T^{*} M$ is an exact symplectic manifold, there is no nonconstant holomorphic sphere $c: S^{2} \rightarrow T^{*} M$. Then, the relation $\left.\omega_{M}\right|_{\pi_{2}\left(T^{*} M, L_{i}\right)}=0$ for $i=1,2$ implies that there is no holomorphic disk $w:(D, \partial D) \rightarrow\left(T^{*} M, L_{i}\right)$. So bubbling does not occur in a sequence in $\mathcal{M}\left(L_{0}, L_{1}\right)$. In this framework, Gromov's classical compactness result about holomorphic curves can be formulated as follows:

Theorem 3.14. For $A>0$ denote $\mathcal{M}_{A}\left(L_{0}, L_{1}\right)$ the space of solutions $v \in \mathcal{M}\left(L_{0}, L_{1}\right)$ with bounded energy $E(v) \leq A$. The space $\mathcal{M}_{A}\left(L_{0}, L_{1}\right)$ is compact in the topology $e_{\mathrm{loc}}^{\infty}$.

As a consequence, we have the corresponding Floer-type compactness result which we will use in the sequel:

Theorem 3.15. For $x, y \in L_{0} \cap L_{1}$ and $A>0$ let

$$
\mathcal{M}_{A}^{*}(x, y)=\left\{v \in \mathcal{M}^{*}(x, y) \mid E(v) \leq A\right\}
$$

Let $\left(v_{n}\right) \subset \mathcal{M}_{A}^{*}(x, y)$ be a sequence of solutions with constant index $\mu\left(v_{n}\right)=\mu_{0}$. Then there exist a finite collection $\left(z_{i}\right)_{i=0, \ldots, k}$ of points in $L_{0} \cap L_{1}$ with $z_{0}=x$ and $z_{k}=y$, some solutions $v^{i} \in \mathcal{M}_{A}^{*}\left(z_{i-1}, z_{i}\right)$ for $i=1, \ldots k$ and some sequences of real numbers $\left(\sigma_{n}^{i}\right)_{n}$ for $i=1, \ldots, k$ such that for all $i=1, \ldots, k$ the sequence $v_{n}\left(s+\sigma_{n}^{i}, t\right)$ converges towards $v^{i}(s, t)$ in $\ell_{\mathrm{loc}}^{\infty}$.

Moreover, we have the relations

$$
\sum_{i=1}^{k} E\left(v^{i}\right) \leq A \quad \text { and } \quad \sum_{i=1}^{k} \mu\left(v^{i}\right)=\mu_{0} .
$$

We say in this case that (modulo a choice of a subsequence) $\left(v_{n}\right)$ converges towards the broken orbit $\left(v^{1}, \ldots, v^{k}\right)$. This theorem is proved in [4], [17] for the Hamiltonian case and stated in [18] for the non-exact one. Since there are some differences between these two situations (for instance, the points $z_{i}$ need not to be different here), we give a complete proof below.

Proof of Theorem 3.15. To simplify the notations, we will denote by $v_{n}(s)$ the path $v_{n}(s, \cdot) \in \Omega\left(L_{0}, L_{1}\right)$.

Denote by $d$ the distance $e^{0}$ on $\Omega\left(L_{0}, L_{1}\right)$, i.e. $d(\alpha, \beta)=\sup _{t} \delta(\alpha(t), \beta(t))$, where $\delta$ is a distance associated to a fixed complete metric on $T^{*} M$. Let $\epsilon>0$ be such that the balls $B(x, \epsilon)$ centered in $x \in L_{0} \cap L_{1}$ are mutually disjoint.

We may also suppose that every nonconstant holomorphic strip $v \in \mathcal{M}^{*}(x, x)$ leaves $B(x, \epsilon)$. This is true for $\epsilon$ small enough. Indeed, the contrary would imply 
that the image of $v$ belongs to a contractible neighbourhood of $x \in T^{*} M$, which means that it lifts to $\bar{v} \in \mathcal{M}(\bar{x}, \bar{x})$ and therefore $E(v)=0$ which is contradictory.

Now denote by $v_{n}\left(\sigma_{n}^{1}\right)$ the point (in $\Omega\left(L_{0}, L_{1}\right)$ ) where the orbit $v_{n}(s)$ first leaves $B(x, \epsilon)$. It is given by

$$
\sigma_{n}^{1}=\inf \left\{s \in \mathbb{R} \mid d\left(x, v_{n}(s)\right)>\epsilon\right\} .
$$

According to Theorem 3.14, there is a subsequence of $v_{n}\left(s+\sigma_{n}^{1}\right)$ which converges in $\bigodot_{\mathrm{loc}}^{\infty}$ to an orbit $v^{1} \in \mathcal{M}\left(L_{0}, L_{1}\right)$. Since $v_{n}\left(\sigma_{n}^{1}\right) \in \partial B(x, \epsilon)$ and for any $s<0$ we have $v_{n}\left(s+\sigma_{n}^{1}\right) \in B(x, \epsilon)$, the limit satisfies $v^{1}(s) \in \bar{B}(x, \epsilon)$ for $s<0$ and $v^{1}(0) \in \partial B(x, \epsilon)$. This implies that $v^{1}$ is a nonconstant solution in $\mathcal{M}\left(x, z_{1}\right)$ (i.e. $\left.v^{1} \in \mathcal{M}^{*}\left(x, z_{1}\right)\right)$ for some $z_{1} \in L_{0} \cap L_{1}$.

Let $s_{*} \in \mathbb{R}$ such that for all $s>s_{*}$ we have $v^{1}(s) \in B\left(z_{1}, \epsilon\right)$. It follows that for $n$ sufficiently large $v_{n}\left(s_{*}+\sigma_{n}^{1}\right)$ is in $B\left(z_{1}, \epsilon\right)$. If the orbit $v_{n}$ does not leave the ball $B\left(z_{1}, \epsilon\right)$ for $s>s_{*}+\sigma_{n}^{1}$, we infer that $z_{1}=y$ and that $v^{1}(s) \in \bar{B}(y, \epsilon)$ for $s \geq s_{*}$. We claim that the proof is finished in this case: we will establish the relations on the energy and on the Maslov index at the end of this proof.

Now if $v_{n}\left(s+s_{*}+\sigma_{n}^{1}\right)$ gets out the ball $B\left(z_{1}, \epsilon\right)$ for some $s>0$ consider the first exit point $v\left(\sigma_{n}^{2}\right)$ defined by

$$
\sigma_{n}^{2}=\sup \left\{\sigma>s_{*}+\sigma_{n}^{1} \mid v_{n}(s) \in B\left(z_{1}, \epsilon\right) \text {, for all } s \in\right] s_{*}+\sigma_{n}^{1} \sigma[\} \text {. }
$$

Using again Theorem 3.14 we find a convergent subsequence of $v_{n}\left(s+\sigma_{n}^{2}\right)$, whose limit is denoted by $v^{2}$.

We want to show that the starting point of $v^{2}$ is $z_{1}$. Remark that $\sigma_{n}^{2}-\sigma_{n}^{1} \rightarrow+\infty$. Indeed, if this sequence was bounded, then on the bounded interval $\left[s_{*}, \sigma_{n}^{2}-\sigma_{n}^{1}\right]$ the sequence $v_{n}\left(s+\sigma_{n}^{1}\right)$ would converge uniformly towards $v^{1}$. In particular $v_{n}\left(\sigma_{n}^{2}\right)$ would be contained in the open ball $B\left(z_{1}, \epsilon\right)$. On the other hand, the definition of $\sigma_{n}^{2}$ implies that $v_{n}\left(\sigma_{n}^{2}\right) \in \partial B\left(z_{1}, \epsilon\right)$, which yields a contradiction.

Fix a number $s<0$. For $n$ sufficiently large we have

$$
\sigma_{n}^{1}+s_{*}<\sigma_{n}^{2}+s<\sigma_{n}^{2} \text {. }
$$

According to the definition of $\sigma_{n}^{2}$, this means that $v_{n}\left(s+\sigma_{n}^{2}\right) \in B\left(z_{1}, \epsilon\right)$ for all $s<0$, therefore, $v^{2}(s) \in \bar{B}\left(z_{1}, \epsilon\right)$ for all $s<0$. On the other hand, obviously $v^{2}(0) \in \partial B\left(z_{1}, \epsilon\right)$. We infer that $v^{2}$ is a non-constant solution in $\mathcal{M}^{*}\left(z_{1}, z_{2}\right)$ for some $z_{2} \in L_{0} \cap L_{1}$.

Then we argue in the same way to find limit solutions $v^{3}, v^{4}, \ldots$ Still we have to prove that this iteration is valid only a finite number of times (which means, as above, that $z_{k}=y$ for some $k$ and that $v_{n}\left(s+\sigma_{n}^{k}\right) \in B(y, \epsilon)$ for $\left.s \geq s_{*}\right)$. Suppose the contrary: there exists a sequence of sequences $\left(\sigma_{n}^{i}\right)$ such that $v_{n}\left(s+\sigma_{n}^{i}\right)$ converges towards $v^{i}(s)$ and moreover $\sigma_{n}^{i+1}-\sigma_{n}^{i}$ are positive and unbounded. Fix a point 
$\bar{z}_{i} \in T^{*} \tilde{M}$ above each $z_{i}$ and lift $v^{i}$ to $T^{*} \tilde{M}$ with $\bar{z}_{i}$ as ending point. The starting point of the lift $\bar{v}^{i}$ will be $g_{i} \bar{z}_{i-1}$ for some $g_{i} \in \pi_{1}(M)$. It follows that

$$
E\left(v_{i}\right)=E\left(\bar{v}_{i}\right)=\mathcal{A}\left(g_{i} \bar{z}_{i-1}\right)-\mathcal{A}\left(\bar{z}_{i}\right)=\mathcal{A}\left(\bar{z}_{i-1}\right)-\mathcal{A}\left(\bar{z}_{i}\right)-u(g) .
$$

This energy is not zero, since $v^{i}$ is non constant, and as $u$ is rational it takes values in a discrete set. Therefore, there is a constant $c>0$ such that

$$
E\left(v^{i}\right)>c \quad \text { for all } i \in \mathbb{N} .
$$

Let us show that $\sum_{i} E\left(v^{i}\right) \leq A$ to get a contradiction. Fix an arbitrary positive $\delta_{i}<E\left(v^{i}\right)$ and real numbers $s_{i}^{-}<s_{i}^{+}$such that

$$
E\left(v^{i}\right)-\delta_{i}=\int_{0}^{1} \int_{s_{i}^{-}}^{s_{i}^{+}}\left\|\partial v^{i} / \partial s\right\|^{2} d s d t .
$$

Due to the $\mathcal{C}_{\mathrm{loc}}^{\infty}$-convergence we have for $n$ sufficiently large (i.e. $n \geq n_{0}$, where $n_{0}$ depends on $\delta_{i}$ ):

$E\left(v^{i}\right)-2 \delta_{i}<\int_{0}^{1} \int_{s_{i}^{-}}^{s_{i}^{+}}\left\|\partial v_{n}\left(s+\sigma_{n}^{i}\right) / \partial s\right\|^{2} d s d t=\int_{0}^{1} \int_{s_{i}^{-}+\sigma_{n}^{i}}^{s_{i}^{+}+\sigma_{n}^{i}}\left\|\partial v_{n}(s) / \partial s\right\|^{2} d s d t$.

Now, for $n$ large enough we have that $s_{i}^{-}+\sigma_{n}^{i}>s_{i-1}^{+}+\sigma_{n}^{i-1}$ and $s_{i}^{+}+\sigma_{n}^{i}<$ $s_{i+1}^{-}+\sigma_{n}^{i+1}$. Summing up the preceding equality for all $i$ we obtain therefore

$$
\sum_{i} E\left(v^{i}\right)-2 \delta_{i}<E\left(v_{n}\right) \leq A .
$$

Put $\delta_{i}=\delta / 2^{i}$, to get $\sum_{i} E\left(v^{i}\right)-\delta \leq A$, so, since $\delta$ is arbitrary, $\sum_{i} E\left(v_{i}\right) \leq A$, which contradicts $E\left(v^{i}\right)>c$ for all $i$.

The required inequality on the energies is obtained as above. It remains to show the relation between the Maslov-Viterbo indices. It is a consequence of the following

Lemma 3.16. Let $\gamma_{n}:[-\infty,+\infty] \rightarrow L_{0}$ the path defined by $\gamma_{n}(s)=v_{n}(s, 0)$ extended with $x$ in $s=-\infty$ and with $y$ in $s=+\infty$. For $i=1, \ldots, k$ let $\gamma^{i}:[-\infty,+\infty] \rightarrow L_{0}$ the analogous paths defined by the holomorphic strips $v^{i}$. Then for $n$ large enough $\gamma_{n}$ and $\gamma=\gamma^{1} * \gamma^{2} * \cdots * \gamma^{k}$ are homotopic in $L_{0}$.

The same is true for the paths defined on $L_{1}$ by $v_{n}, v^{1}, \ldots, v^{k}$.

Proof. For $i=1, \ldots, k$ consider the sequences $\left(\sigma_{n}^{i}\right)$ defined in the proof of Theorem 3.15 above. They satisfy the following properties for some $s_{*}>0$ :

- $v_{n}(s) \in B(x, \epsilon)$ for $s \leq \sigma_{n}^{1}$. 
- $v_{n}(s) \in B\left(z_{i}, \epsilon\right)$ for $s \in\left[s_{*}+\sigma_{n}^{i}, \sigma_{n}^{i+1}\right]$.

- $v_{n}(s) \in B(y, \epsilon)$ for $s \geq s_{*}+\sigma_{n}^{k}$.

- $v^{i}(s) \in B\left(z_{i-1}, \epsilon\right)$ for $s \leq 0$, for all $i=1, \ldots, k$.

- $v^{i}(s) \in B\left(z_{i}, \epsilon\right)$ for $s \geq s_{*}$, for all $i=1, \ldots, k$.

- $v_{n}\left(s+\sigma_{n}^{i}\right)$ converges towards $v^{i}(s)$ uniformly on $\left[0, s_{*}\right]$ (and more generally on every compact interval).

The $\epsilon$-balls above are defined by the $\bigodot^{0}$-distance $d$ on $\Omega\left(L_{0}, L_{1}\right)$ corresponding to a complete metric on $T^{*} M$. Consider complete metrics on $L_{0}$ and $L_{1}$, with associated distances $d_{0}$ and $d_{1}$. Define the distance $d^{\prime}$ on $\Omega\left(L_{0}, L_{1}\right)$ by the formula

$$
d^{\prime}(\alpha, \beta)=\max \left\{d(\alpha, \beta), d_{0}(\alpha(0), \beta(0)), d_{1}(\alpha(1), \beta(1))\right\} .
$$

It is easy to see that we can write the first part of the proof of Theorem 3.15 for this new distance, so we may suppose that the properties of $v_{n}$ and $v^{i}$ above are valid for the $\epsilon$-balls defined by $d^{\prime}$. In particular, we can replace $v_{n}$ by $\gamma_{n}, v^{i}$ by $\gamma^{i}$ and the distance by $d_{0}$.

Now let $\chi: L_{0} \rightarrow L_{0}$ be a continuous map which is homotopic to the identity and satisfies $\chi\left(B\left(z_{i}, \epsilon\right)\right)=z_{i}$ for all $i=1, \ldots, k$. Obviously $\gamma_{n}^{\prime}=\chi\left(\gamma_{n}\right)$ is homotopic to $\gamma_{n}$ and $\gamma^{\prime}=\chi(\gamma)$ is homotopic to $\gamma$. Let us show that $\gamma_{n}^{\prime}$ is homotopic to $\gamma^{\prime}$ for $n$ large enough. We know that $\gamma_{n}\left(s+\sigma_{n}^{1}\right)$ converges towards $\gamma^{1}(s)$ uniformly on $\left[0, s_{*}\right]$. Therefore, for $n$ sufficiently large, $\gamma_{n}\left(\cdot+\sigma_{n}^{1}\right)$ and $\gamma^{1}$ are homotopic in $L_{0}$. To see this, one has to write

$$
\gamma_{n}\left(s+\sigma_{n}^{1}\right)=\exp _{\gamma^{1}(s)} Y_{n}(s)
$$

for some $Y_{n}:\left[0, s_{*}\right] \rightarrow\left(\gamma^{1}\right)^{*} T L_{0}$, and then to consider the homotopy

$$
\lambda \mapsto \exp _{\gamma^{1}(s)} \lambda Y_{n}(s) .
$$

It follows that $\chi\left(\gamma_{n}\left(\cdot+\sigma_{n}^{1}\right)\right)$ and $\chi\left(\gamma^{1}\right)$ are homotopic as paths defined on $\left[0, s_{*}\right]$. In the same way $\chi\left(\gamma_{n}\left(\cdot+\sigma_{n}^{i}\right)\right)$ and $\chi\left(\gamma^{i}\right)$ are homotopic for all $i=1, \ldots, k$. Summarizing, we have for $n$ sufficiently large:

- $\gamma_{n}^{\prime}(s)=z_{i}$ for $s \in\left[s_{*}+\sigma_{n}^{i}, \sigma_{n}^{i+1}\right]$ for $i=1, \ldots, k-1 ; \gamma_{n}^{\prime}(s)=x$ for $s \leq \sigma_{n}^{1}$; $\gamma_{n}^{\prime}(s)=y$ for $s \geq s_{*}+\sigma_{n}^{k}$.

- $\chi\left(\gamma^{i}(s)\right)=z_{i-1}$ for $s \in[-\infty, 0]$ for $i=1, \ldots, k ; \chi\left(\gamma^{i}(s)\right)=z_{i}$ for $s \in$ $\left[s_{*},+\infty[\right.$ for $i=1, \ldots, k$.

- $\gamma_{n}^{\prime}\left(s+\sigma_{n}^{i}\right)$ and $\chi\left(\gamma^{i}(s)\right)$ are homotopic on $s \in\left[0, s_{*}\right]$ for $i=1, \ldots, k$.

One easily infers that for an appropriate parametrisation $\gamma_{n}^{\prime}$ and $\gamma^{\prime}=\chi\left(\gamma^{1} * \gamma^{2} *\right.$ $\cdots * \gamma^{k}$ ) are homotopic. The lemma is proved (the argument for the paths on $L_{1}$ is completely analogous). 
Now we are able to finish the proof of Theorem 3.15. In [25], C. Viterbo proved the following

Theorem 3.17. Let $v, w \in \mathcal{M}(x, y)$. Consider the paths $\gamma_{i}^{v}:[-\infty,+\infty] \rightarrow L_{i}$ and $\gamma_{i}^{w}:[-\infty,+\infty] \rightarrow L_{i}$ defined by the restrictions of $v$ and $w$ to $\mathbb{R} \times\{i\}$, for $i=0,1$. Then

$$
\mu(w)-\mu(v)=\mu_{L_{1}}\left(\gamma_{1}^{w} *\left(\gamma_{1}^{v}\right)^{-1}\right)-\mu_{L_{0}}\left(\gamma_{0}^{w} *\left(\gamma_{0}^{v}\right)^{-1}\right),
$$

where $\mu_{L_{i}}$ is the Maslov class of the Lagrangian manifold $L_{i}$ for $i=0,1$.

We apply the previous statement to the strips $v_{n}$ and $v^{1} \# \cdots \# v^{k}$. The MaslovViterbo index of the latter is obviously $\sum_{i} \mu\left(v^{i}\right)$. Then, by Lemma 3.16, the loops in the right term of the relation above are null homotopic for $n$ sufficiently large, so this term actually vanishes for large $n$. Therefore

$$
\mu_{0}=\sum_{i=1}^{k} \mu\left(v^{i}\right),
$$

and the proof of the theorem is finished.

3.5. The differential of the Floer complex. Let $x, y \in L_{0} \cap L_{1}$. We define an "incidence number" $[x, y]$ like in Novikov theory (Section 2.2). We proceed as follows. Denote by $\mathscr{L}^{0}(x, y)$ the zero-dimensional component of $\mathscr{L}(x, y)=$ $\mathcal{M}^{*}(x, y) / \mathbb{R}$. For any $z \in L_{0} \cap L_{1}$ fix a lift $\bar{z} \in T^{*} \tilde{M}$. For $g \in \pi_{1}(M)$, consider the subset $\mathscr{L}_{g}^{0}(x, y) \subset \mathscr{L}^{0}(x, y)$ of trajectories which lift to $\mathscr{L}(g \bar{x}, \bar{y})$. We need the following

Lemma 3.18. Under the assumptions of Theorem 3.12, for any $x, y \in L_{0} \cap L_{1}$ and $g \in \pi_{1}(M)$ the set $\mathscr{L}_{g}^{0}(x, y)$ is finite.

If $n_{g} \in \mathbb{Z} / 2 \mathbb{Z}$ denotes its parity, then the sum $\sum n_{g} g$ belongs to the Novikov ring $\Lambda_{-u}$.

Proof. The elements of $\mathscr{L}_{g}^{0}(x, y)$ are classes of solutions $v$ which belong to the 1-dimensional component of $\mathcal{M}^{*}(x, y)$. Moreover these solutions have the same energy:

$$
E(v)=\mathcal{A}(\bar{x})-\mathcal{A}(\bar{y})-u(g) .
$$

We can therefore apply Theorem 3.15. Since all the manifolds $\mathcal{M}^{*}(x, y)$ have dimensions greater than one (because of the free action of $\mathbb{R}$ ), a sequence of solutions $v_{n}$ of Maslov index $\mu=1$ cannot converge towards a broken orbit. Therefore, it admits a subsequence which converges in the sense of Theorem 3.15 towards a solution in $\mathcal{M}^{*}(x, y)$. This means that $\mathscr{L}_{g}^{0}(x, y)$ is compact, therefore it has a finite number of elements. 
Let us now prove that $\sum n_{g} g \in \Lambda_{-u}$. Let $C<0$. Our claim is proved if we show that the set $\bigcup_{u(g) \geq C} \mathscr{L}_{g}^{0}(x, y)$ has finite cardinality. It suffices to show that it is compact. A sequence $\left(w_{n}\right)$ in this space lifts to a sequence $\left(v_{n}\right)$ in an 1-dimensional component of $\mathcal{M}^{*}(g \bar{x}, \bar{y})$. If $u(g) \geq C$, then the energy of the solutions $v$ above satisfy

$$
E\left(v_{n}\right) \leq \mathcal{A}(\bar{x})-\mathcal{A}(\bar{y})-C .
$$

The sequence $\left(w_{n}\right)$ is therefore contained in $\mathcal{M}_{A}^{*}(x, y)$ where $A>0$ is given by the right term above. As above, none of its subsequences converges towards a splitting orbit. Therefore, by Theorem 3.15, $\left(v_{n}\right)$ admits a subsequence which is convergent in the considered 1-dimensional component of $\mathcal{M}_{A}^{*}(x, y)$ (in the sense of Theorem 3.15), which means that a subsequence of $\left(w_{n}\right)$ converges in $\mathscr{L}^{0}(x, y)$. Moreover, the relation between energies in Theorem 3.15 shows that the limit lies in $\bigcup_{u(g) \geq C} \mathscr{L}_{g}^{0}(x, y)$. This space is therefore compact and zero dimensional, i.e. of finite cardinality.

We define

$$
[x, y]=\sum_{g \in \pi_{1}(M)} n_{g}(x, y) g,
$$

where $n_{g}(x, y)=\# \mathscr{L}_{g}^{0}(x, y)$ as above. Then, we consider the complex

$$
C_{\bullet}\left(L_{0}, L_{1}, J_{t}\right)=\Lambda_{-u}\left\langle L_{0} \cap L_{1}\right\rangle,
$$

with differential

$$
\partial x=\sum_{y \in L_{0} \cap L_{1}}[x, y] y=\sum_{\substack{g \in \pi_{1}(M) \\ y \in L_{0} \cap L_{1}}} n_{g}(x, y) g y .
$$

To show the relation $\partial^{2}=0$ we have to prove that for each $g \in \pi_{1}(M)$ and $x, z \in L_{0} \cap L_{1}$ we have

$$
\sum_{\substack{y \in L_{0} \cap L_{1}, g^{\prime}, g^{\prime \prime} \in \pi_{1}(M) \\ g^{\prime \prime} g^{\prime}=g}} n_{g^{\prime}}(x, y) n_{g^{\prime \prime}}(y, z)=0 .
$$

This is a straightforward consequence of

Lemma 3.19. Let $\mathscr{L}_{g}^{1}(x, z)$ be the 1-dimensional component of $\mathscr{L}_{g}(x, z)$. Denote by $\overline{\mathscr{L}}_{g}^{1}(x, z)$ the union

$$
\mathscr{L}_{g}^{1}(x, z) \cup \bigcup_{\substack{y \in L_{0} \cap L_{1} \\ g^{\prime \prime} g^{\prime}=g}} \mathscr{L}_{g^{\prime}}^{0}(x, y) \times \mathscr{L}_{g^{\prime \prime}}^{0}(y, z),
$$


endowed with the topology given by the convergence towards broken orbits which was defined in Theorem 3.15.

Then $\mathscr{L}_{g}^{1}(x, z)$ is a compact 1-dimensional manifold whose boundary is

$$
\bigcup_{\substack{y \in L_{0} \cap L_{1} \\ g^{\prime \prime} g^{\prime}=g}} \mathscr{L}_{g^{\prime}}^{0}(x, y) \times \mathscr{L}_{g^{\prime \prime}}^{0}(y, z) .
$$

Sketch of the proof. To prove the compactness, let $\left(w_{n}\right)$ be a sequence in $\mathscr{L}_{g}^{1}(x, z)$. It admits a lift $\left(v_{n}\right) \in \mathcal{M}^{*}(x, z)$ such that $\mu\left(v_{n}\right)=2$. By applying Theorem 3.15 we find that modulo the choice of a subsequence $\left(v_{n}\right)$ converges either towards a limit $v^{0} \in \mathcal{M}^{*}(x, z)$ or towards a broken orbit $\left(v^{1}, v^{2}\right) \in \mathcal{M}^{*}(x, y) \times \mathcal{M}^{*}(y, z)$ for some $y \in L_{0} \cap L_{1}$. For $i=0,1,2$ we denote by $w^{i}$ the projections of $v^{i}$ on the correspondent trajectory spaces $\mathscr{L}$. In the first case, we infer using also Lemma 3.16 that (modulo the choice of a subsequence) $\left(w^{n}\right)$ converges towards $w^{0} \in \mathscr{L}_{g}^{1}(x, z)$. In the last case we obtain using again Lemma 3.16 that $\left(w_{n}\right)$ converges towards $\left(w^{1}, w^{2}\right) \in \mathscr{L}_{g^{\prime}}^{0}(x, y) \times \mathscr{L}_{g^{\prime \prime}}^{0}(y, z)$ where $g^{\prime}, g^{\prime \prime} \in \pi_{1}(M)$ satisfy $g^{\prime \prime} g^{\prime}=g$.

Conversely, starting with $\left(w^{1}, w^{2}\right) \in \mathscr{L}_{g^{\prime}}^{0}(x, y) \times \mathscr{L}_{g^{\prime \prime}}^{0}(y, z)$ one may use the usual gluing argument [5] to get a parametrisation $\Psi:\left[0,1\left[\rightarrow \mathscr{L}^{1}(x, z)\right.\right.$ which satisfies $\lim _{\rho \rightarrow 1} \Psi(\rho)=\left(w^{1}, w^{2}\right)$. Using Lemma 3.16 we obtain that the image of $\Psi$ is contained in the component $\mathscr{L}_{g^{\prime \prime} g^{\prime}}^{1}(x, z)=\mathscr{L}_{g}^{1}(x, z)$.

Remark 3.20. In a similar manner one can define a complex $C_{\bullet}(L, L)$ which is spanned by the zeroes of the 1 -form $\hat{v}$, i.e. the trajectories of the flow of $X_{t}$ starting in $L$ for $t=0$ and ending in $L$ for $t=1$. To define the differential one has to use the solution spaces $\widehat{\mathcal{M}}(x, y)$ defined by $(* *)$ in Section 3.3. Recall that in the relation in Lemma 3.8 written for $\hat{\mathcal{A}}$ one has to change $-u$ in $u$. This means that the complex $C_{\bullet}(L, L)$ is defined over the Novikov ring $\Lambda_{u}$. We will denote it by $C_{\bullet}\left(L, \phi_{t}, J_{t}\right)$ to emphasize its dependence on the symplectic isotopy and on the almost complex structure. Using the correspondence from Remark 3.11 one easily infers that the $\Lambda_{u}$-complexes $C_{\bullet}\left(L, \phi_{t}, \hat{J}_{t}\right)$ and $C_{\bullet}\left(L_{0}, \phi_{1}^{-1}\left(L_{0}\right), J_{t}\right)$ are actually isomorphic.

In the next section we will only consider the complex $C_{\bullet}(L, L)=C_{\bullet}\left(L, \phi_{t}, J_{t}\right)$.

3.6. Hamiltonian invariance. We denote by $H_{*}\left(L, \phi_{t}, J_{t}\right)$ the homology of $C_{\bullet}\left(L, \phi_{t}, J_{t}\right)$. Recall that the symplectic isotopy $\left(\phi_{t}\right)$ is supposed to be defined by $\alpha+d H_{t}$, with $\alpha$ closed 1-form on $M$ and $H$ compactly supported on $T^{*} M \times[0,1]$. We want to show that this homology does not depend on a generic choice of the couple $\left(J_{t}, H_{t}\right)$ which means that it only depends on $L$ and on the cohomology class $[\alpha]$ (see the analogous result for periodic orbits in [14], Theorem 4.3): 
Theorem 3.21. For generic pairs $\left(H_{t}, J_{t}\right),\left(H_{t}^{\prime}, J_{t}^{\prime}\right)$ there is an isomorphism

$$
\Psi: H_{*}\left(L, \phi_{t}^{\alpha+d H_{t}}, J_{t}\right) \rightarrow H_{*}\left(L, \phi_{t}^{\alpha+d H_{t}^{\prime}}, J_{t}^{\prime}\right) .
$$

Proof. The proof is similar to the one in [14], following the standard arguments in [4] and [11]. We consider a family of functions $H_{s, t}: T^{*} M \rightarrow \mathbb{R}$ and a family of compatible complex structures $J_{s, t}$ which depend smoothly on $(s, t) \in \mathbb{R}^{2}$ and which satisfy $\left(H_{s, t}, J_{s, t}\right)=\left(H_{t}, J_{t}\right)$ for $s<-R$ and $\left(H_{s, t}, J_{s, t}\right)=\left(H_{t}^{\prime}, J_{t}^{\prime}\right)$ for $s>R$, where $R>0$ is fixed. In order to define $\Psi$ we consider the space $\mathcal{M}_{H_{s, t}, J_{s, t}}(L)$ defined by

$$
\left\{v: \mathbb{R} \times[0,1] \rightarrow T^{*} M|| \begin{array}{c}
\frac{\partial v}{\partial s}+J_{t}\left(\frac{\partial v}{\partial t}-X_{s, t}^{\alpha+d H_{s, t}}\right)=0, \\
v(s, i) \in L \text { for } i=1,2, s \in \mathbb{R},
\end{array} E(v)<+\infty\right\}
$$

The restrictions of an element $v$ of $\mathcal{M}_{H_{s, t}, J_{s, t}}(L)$ to $s<-R$ resp. to $s>R$ are solutions of $(* *)$ corresponding to the couples $\left(H_{t}, J_{t}\right)$ resp. $\left(H_{t}^{\prime}, J_{t}^{\prime}\right)$. One can then infer the analogue of Theorem 3.10, namely the fact that any such $v$ converges towards a zero $x$ of the action 1 -form $\hat{v}$ when $s$ tends to $-\infty$ and towards a zero $y$ of the action 1-form $\hat{v}^{\prime}$ (corresponding to the Hamiltonian $H_{t}^{\prime}$ ) when $s$ tends to $+\infty$. Therefore $\mathcal{M}_{H_{S, t}, J_{s, t}}(L)$ is the union of the spaces $\mathcal{M}_{H_{s, t}, J_{s, t}}(x, y)$ given by

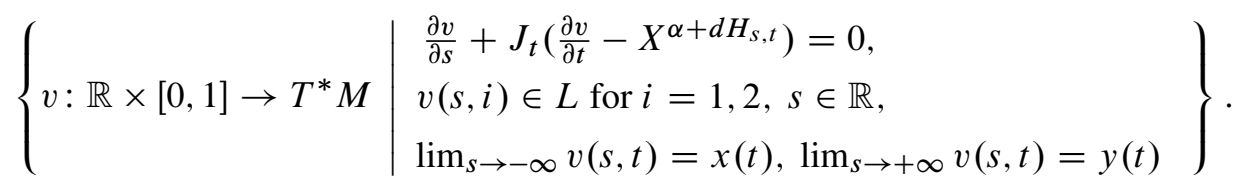

Here $x, y$ are zeroes of the action 1-forms $\hat{v}$ resp. $\hat{v}^{\prime}$. The analogue of Theorem 3.12 is valid: For a generic choice of the couple $\left(H_{s, t}, J_{s, t}\right)$ the spaces $\mathcal{M}_{H_{s, t}, J_{s, t}}(x, y)$ are manifolds of local dimension at $v$ equal to the Maslov-Viterbo index $\mu(v)$. We will define a morphism of $\Lambda_{u}$ - complexes

$$
\Psi_{\bullet}: C_{\bullet}\left(L, \phi_{t}^{\alpha+d H_{t}}, J_{t}\right) \rightarrow C_{\bullet}\left(L, \phi_{t}^{\alpha+d H_{t}^{\prime}}, J_{t}^{\prime}\right) .
$$

On the prescribed generators of $C_{\bullet}\left(L, \phi_{t}^{\alpha+d H_{t}}, J_{t}\right)$ it is given by the formula

$$
\Psi_{\bullet}(x)=\sum_{g \in \pi_{1}(M), y} m_{g}(x, y) g y,
$$

where $m_{g}(x, y) \in \mathbb{Z} / 2 \mathbb{Z}$ will be defined below. For this purpose we have to consider the zero-dimensional components $\mathcal{M}_{H_{s, t}, J_{s, t}}^{0}(x, y)$. To count the elements of $\mathcal{M}^{0}$ we need a compactness result analogous to Theorem 3.15. We obtain indeed as in Theorem 3.15 that any sequence $\left(v_{n}\right)$ in

$$
\mathcal{M}_{H_{s, t}, J_{s, t}}(x, y ; A)=\left\{v \in \mathcal{M}_{H_{s, t}, J_{s, t}}(x, y) \mid E(v) \leq A\right\}
$$


has a subsequence which converges towards a broken orbit $\left(v^{1}, v^{2}, \ldots, v^{k}\right)$. Actually, if the homotopy $\left(H_{s, t}, J_{s, t}\right)_{s}$ is not trivial, only precisely one $v^{i}$ in the limit belongs to $\mathcal{M}_{H_{S, t}, J_{s, t}}(L)$; the preceding orbits $v^{1}, \ldots, v^{i-1}$ are in $\mathcal{M}_{H_{t}, J_{t}}(L)$ and the last ones $v^{i+1}, \ldots, v^{k}$ are in $\mathcal{M}_{H_{t}^{\prime}, J_{t}^{\prime}}(L)$. More precisely, for $j=1, \ldots, k, v^{j}$ is the limit of $v_{n}\left(\cdot+\sigma_{n}^{j}, \cdot\right)$ where $\sigma_{n}^{i}=0$ and the sequences $\left(\sigma_{n}^{j}\right)$ tend to $-\infty$ for $j<i$, resp. to $+\infty$ for $j>i$. (Too see this one has just to pass to the limit in the Floer equation which defines $\mathcal{M}_{H_{s, t}, J_{s, t}}(L)$.) The energy and the Maslov index of the limit satisfy the same relations as in Theorem 3.15.

As a consequence, we have

Lemma 3.22. For any $A>0$ the set $\mathcal{M}_{H_{S, t}, J_{s, t}}^{0}(x, y ; A)$ is finite.

Proof. Any sequence $\left(v_{n}\right) \in \mathcal{M}_{H_{s, t}, J_{s, t}}^{0}(x, y ; A)$ has a subsequence which converges towards a broken orbit $\left(v^{1}, \ldots, v^{k}\right)$ as above. It follows that $k=0$, since nonconstant orbits in $\mathcal{M}_{H_{t}, J_{t}}(L)$ and in $\mathcal{M}_{H_{t}^{\prime}, J_{t}^{\prime}}(L)$ have non zero Maslov-Viterbo indices. Therefore the space $\mathcal{M}_{H_{s, t}, J_{s, t}}^{0}(x, y ; A)$ is compact and zero-dimensional, so it is finite.

As in the preceding subsection, fix a lift $\bar{x}$ in $T^{*} \tilde{M}$ of every zero $x$ of the action 1 -form $\hat{v}$ and a lift $\bar{y}$ in $T^{*} \tilde{M}$ for every zero $y$ of $\hat{v}^{\prime}$. Consider for $g \in \pi_{1}(M)$ and any two zeroes $x, y$ of $\hat{v}$ resp. $\hat{v}^{\prime}$ the space

$$
\mathcal{M}_{g, s}(x, y) \subset \mathcal{M}_{H_{s, t}, J_{s, t}}(x, y),
$$

consisting of the orbits which lift to $T^{*} \tilde{M}$ starting from $g \bar{x}$ and ending at $\bar{y}$. The following proposition is crucial for the proof of Theorem 3.21

Proposition 3.23. The space $\mathcal{M}_{g, s}(x, y)$ is contained in $\mathcal{M}_{H_{s, t}, J_{s, t}}(x, y ; A)$ for some $A>0$.

Proof. We adapt the standard argument of [5] as in [14] (see also [4], [11]). Let $v \in \mathcal{M}_{g, s}(x, y)$. We find an upper bound for $E(v)$. Note that in the inequalities below (and actually in the definition of the energy) the norm is defined by the compatible metric $\omega_{M}\left(\cdot, J_{s, t}(\cdot)\right)$ (it therefore depends on $(s, t)$ ):

$$
\begin{aligned}
E(v) & =\int_{\mathbb{R} \times[0,1]}\left\|\frac{\partial v}{\partial s}\right\| d s d t=\int_{\mathbb{R} \times[0,1]} \omega_{M}\left(\frac{\partial v}{\partial s}, J_{s, t} \frac{\partial v}{\partial s}\right) d s d t \\
& =\int_{\mathbb{R} \times[0,1]} \omega_{M}\left(\frac{\partial v}{\partial s}, \frac{\partial v}{\partial t}-X^{\alpha}-X^{d H_{s, t}}\right) d s d t \\
& =\int_{v} \omega_{M}-\int_{\mathbb{R} \times[0,1]}\left(\alpha+d H_{s, t}\right)\left(\frac{\partial v}{\partial s}\right) d s d t
\end{aligned}
$$


We see $v:[-\infty,+\infty] \times[0,1]$ as a path in $\Omega(L, L)$ between $x$ and $y$. Fix $z_{0} \in$ $\Omega(L, L)$ and let $w$ be a fixed path in $\Omega(L, L)$ which joins $y$ and $z_{0}$. Denote by $v \# w$ the concatenation of $v$ and $w$. The path $w$ lifts to a path in $T^{*} \tilde{M}$ joining $\bar{y}$ and $\bar{z}_{0}$. Denote by $\hat{\mathcal{A}}$ and $\hat{\mathcal{A}}^{\prime}$ the primitives of $\left(\pi^{\Omega}\right)^{*} v$ resp. of $\left(\pi^{\Omega}\right)^{*} \nu^{\prime}$ which vanish in $\bar{z}_{0}$. We have

$$
\int_{v \# w} \hat{v}-\int_{w} \hat{v}^{\prime}=\left(\hat{\mathcal{A}}\left(\bar{z}_{0}\right)-\hat{\mathcal{A}}(g \bar{x})\right)-\left(\hat{\mathcal{A}}^{\prime}\left(\bar{z}_{0}\right)-\hat{\mathcal{A}}^{\prime}(\bar{y})\right)=-\hat{\mathcal{A}}(g \bar{x})+\hat{\mathcal{A}}^{\prime}(\bar{y}) .
$$

On the other hand, as in the computation in the proof of Lemma 3.8 at the end of Section 3.2, we have

$$
\begin{aligned}
& \int_{v \# w} \hat{v}-\int_{w} \hat{v}^{\prime} \\
& =-\int_{v \# w} \omega_{M}+\int_{\mathbb{R} \times[0,1]}\left(\alpha+d H_{t}\right)\left(\frac{\partial w}{\partial s}\right) d s d t \\
& \quad+\int_{\mathbb{R} \times[0,1]}\left(\alpha+d H_{t}\right)\left(\frac{\partial v}{\partial s}\right) d s d t+\int_{w} \omega_{M}-\int_{\mathbb{R} \times[0,1]}\left(\alpha+d H_{t}^{\prime}\right)\left(\frac{\partial w}{\partial s}\right) d s d t \\
& =-\int_{v} \omega_{M}+\int_{\mathbb{R} \times[0,1]} \frac{\partial}{\partial s}\left(H_{t}(w)+H_{t}(v)-H_{t}^{\prime}(w)\right) d s d t+\int_{\mathbb{R} \times[0,1]} \alpha\left(\frac{\partial v}{\partial s}\right) d s d t \\
& =-\int_{v} \omega_{M}+\int_{[0,1]} H_{t}\left(z_{0}\right)-H_{t}(x)-H_{t}^{\prime}\left(z_{0}\right)+H_{t}^{\prime}(y) d t+\int_{\mathbb{R} \times[0,1]} \alpha\left(\frac{\partial v}{\partial s}\right) d s d t .
\end{aligned}
$$

Denote by $C$ the term $\int_{[0,1]} H_{t}\left(z_{0}\right)-H_{t}^{\prime}\left(z_{0}\right) d t$ which does not depend on $v$. Using (3) we get

$$
\begin{aligned}
& \hat{\mathcal{A}}(g \bar{x})-\hat{\mathcal{A}}^{\prime}(\bar{y}) \\
& =-C+\int_{v} \omega_{M}-\int_{\mathbb{R} \times[0,1]} \alpha\left(\frac{\partial v}{\partial s}\right) d s d t-\int_{[0,1]}-H_{t}(x)+H_{t}^{\prime}(y) d t \\
& =-C+\int_{v} \omega_{M}-\int_{\mathbb{R} \times[0,1]} \alpha\left(\frac{\partial v}{\partial s}\right) d s d t-\int_{\mathbb{R} \times[0,1]} \frac{\partial}{\partial s} H_{s, t}(v) d s d t \\
& =-C+\int_{v} \omega_{M}-\int_{\mathbb{R} \times[0,1]}\left(\alpha+d H_{s, t}\right)\left(\frac{\partial v}{\partial s}\right) d s d t-\int_{\mathbb{R} \times[0,1]} \frac{\partial H}{\partial s}(s, t, v) d s d t \\
& =-C+E(v)-\int_{\mathbb{R} \times[0,1]} \frac{\partial H}{\partial s}(s, t, v) d s d t
\end{aligned}
$$

by the equality (2).

The equality (4) implies that

$$
E(v)=C+\hat{\mathcal{A}}(g \bar{x})-\hat{\mathcal{A}}^{\prime}(\bar{y})+\int_{\mathbb{R} \times[0,1]} \frac{\partial H}{\partial s}(s, t, v) d s d t .
$$


Since $\partial H / \partial s: \mathbb{R} \times[0,1] \times T^{*} M \rightarrow \mathbb{R}$ has compact support, we infer that

$$
E(v) \leq \hat{\mathcal{A}}(g \bar{x})-\hat{\mathcal{A}}^{\prime}(\bar{y})+K=\hat{\mathcal{A}}(\bar{x})-\hat{\mathcal{A}}^{\prime}(\bar{y})+u(g)+K,
$$

for some $K$ which does not depend on $v$. It follows that $\mathcal{M}_{g, s}(x, y)$ is contained in $\mathcal{M}_{H_{S, t}, J_{S, t}}(x, y ; A)$ for some $A>0$, as required.

A straightforward consequence of Proposition 3.23 is that the set

$$
\mathcal{M}_{g, s}^{0}(x, y)=\mathcal{M}_{g, s}(x, y) \cap \mathcal{M}_{H_{s, t}, J_{s, t}}^{0}(x, y)
$$

has finite cardinality. This enables us to define the morphism

$$
\Psi_{\bullet}: C_{\bullet}\left(L, \phi_{t}^{\alpha+d H_{t}}, J_{t}\right) \rightarrow C_{\bullet}\left(L, \phi_{t}^{\alpha+d H_{t}^{\prime}}, J_{t}^{\prime}\right)
$$

by the formula

$$
\Psi_{\bullet}(x)=\sum_{g \in \pi_{1}(M), y} m_{g}(x, y) g y,
$$

where $m_{g}(x, y)$ is the parity of $\mathcal{M}_{g, s}^{0}(x, y)$. Note that by Proposition 3.23 the coefficients $\sum_{g \in \pi_{1}(M)} m_{g}(x, y) g$ belong to $\Lambda_{u}$. Indeed, for any $B \in \mathbb{R}$, the inequality (5) above shows that $\bigcup_{u(g)<B} \mathcal{M}_{g, s}^{0}(x, y)$ is contained in $\mathcal{M}_{H_{s, t}, J_{s, t}}^{0}(x, y ; A)$ for some positive constant $A$, so it is finite, according to Lemma 3.18.

The fact that $\Psi$. commutes with the differentials can be proved in the usual way, by studying the compactness of the 1-dimensional components of $\mathcal{M}_{H_{s, t}, J_{s, t}}(x, y)$ like in the proof of Theorem 3.15. (a sequence in this space either admits a convergent subsequence, or converges towards a broken orbit $\left.\left(v^{1}, v^{2}\right)\right)$. The proof is similar to Lemma 3.19.

Finally, to show that $\Psi$. induces an isomorphism in homology, one again uses the standard method of Floer theory from Theorem 3.15 (construct a morphism $\Gamma_{\bullet}: C_{\bullet}\left(L, \phi_{t}^{\alpha+d H_{t}^{\prime}}, J_{t}^{\prime}\right) \rightarrow C_{\bullet}\left(L, \phi_{t}^{\alpha+d H_{t}}, J_{t}\right)$ and than prove that $\Psi_{\bullet} \Gamma_{\bullet}$ and $\Gamma_{\bullet} \Psi_{\bullet}$ are homotopic to the identity, using a two-parameter homotopy $H_{r, s, t}$ ).

The proof of Theorem 3.21 is now finished.

Remark 3.24. The complex $C_{\bullet}\left(L, \phi_{t}, J_{t}\right)$ defined in the previous subsection is free over the Novikov ring $\Lambda_{-u}$. As we noted in Remark 3.20 one can infer the existence of a similar complex over $\Lambda_{u}$. We showed above that the homology of these complexes only depends on $L$ and on $u$. But the goal of our theorem 3.1 was more general, namely the existence of a complex which is free over $\Lambda_{p^{*} u}$ (spanned by the intersection points $\left.L \cap \phi_{t}(L)\right)$. In the next subsection we show how to adapt the previous construction in order to get this conclusion. 
3.7. The Floer-Novikov complex over $\Lambda_{p^{*} \boldsymbol{u}}$. The idea is the following. Consider the intersection points $L \cap \phi_{1}(L)$, viewed as points in $L$. For two such points $x, y$, any holomorphic strip $v \in \mathcal{M}(x, y)$ defines a path $\gamma:]-\infty,+\infty[\rightarrow L$ which joins $x$ and $y$ :

$$
\gamma(s)=v(s, 0) .
$$

Look at the collection of intersection points and take the paths $\gamma$ as above, defined by the strips $v$ which belong to the one-dimensional component of $\mathcal{M}(x, y)$ (which corresponds to the zero dimensional component of $\mathscr{L}(x, y)$ ). This collection of points and paths joining them is sufficient to re-construct the complex $C_{\bullet}\left(L, \phi_{t}, J_{t}\right)$ : one just has to fix lifts $\bar{x} \in \bar{L}$ for any point and then lift the lines $\gamma$ from $g \bar{x}$ to $\bar{y}$. We get thus the same "incidence number" $[x, y] \in \Lambda_{-u}$ as above. Therefore we obtain the same complex.

Now, instead of lifting these lines to the covering space $\bar{L}$, we lift them to the universal cover $\tilde{L}$. If we start with fixed lifts $\tilde{x} \in \tilde{L}$ of the intersection points, we get thus a new incidence number $[x, y]^{\sim}$ which belongs to the Novikov ring $\Lambda_{-p^{*} u}$. This enables us to define the desired complex.

This idea can be formalized as follows: Let $L$ be a closed manifold and let $\mathcal{C}$ be a finite set of points on $L$. Consider a (possibly infinite) collection $\mathscr{E}$ of paths $\gamma:[-\infty,+\infty] \rightarrow L$ such that $\gamma( \pm \infty) \in \mathcal{C}$. Let $p: \pi_{1}(L) \rightarrow G$ be an epimorphism onto a group $G$ and let $u: G \rightarrow \mathbb{Z}$ be a group morphism. Consider the covering space $\bar{L} \rightarrow L$ associated to $\operatorname{Ker}(p) \subset \pi_{1}(L)$. For any $x \in \mathcal{C}$ fix a lift $\bar{x} \in \bar{L}$. For $x, y \in \mathcal{C}$, and $g \in G$, denote $\mathscr{L}_{g}^{0}(x, y)$ the set of the paths $\gamma \in \mathscr{E}$ which have a lift in $\bar{L}$ which joins $g \bar{x}$ and $\bar{y}$. Denote by $\Lambda_{u}$ the Novikov $\operatorname{ring} \mathbb{Z} / 2[G]_{u}$. Now we prove:

Proposition 3.25. Suppose that:

(a) For any $x, y \in \mathcal{C}$ and $g \in G$ the space $\mathscr{L}_{g}^{0}(x, y)$ is finite and

$$
[x, y]=\sum_{g \in G} \#_{2} \mathscr{L}_{g}^{0}(x, y) g \in \Lambda_{-u} .
$$

(b) The formula

$$
\partial x=\sum_{y \in \mathcal{C}}[x, y] y
$$

defines the differential of a $\Lambda_{-u}$-free complex $C_{\bullet}$ spanned by $\mathcal{C}$. Note that this is equivalent to the fact that for any $x, z \in \mathcal{C}$ and $g \in G$ the space

$$
\bigcup_{y \in \mathcal{C}, g^{\prime \prime} g^{\prime}=g} \mathscr{L}_{g^{\prime}}^{0}(x, y) \times \mathscr{L}_{g^{\prime \prime}}^{0}(y, z)
$$

has an even number of elements. 
(c) The space above is a disjoint union of sets with two elements $\left\{\left(\gamma_{1}, \gamma_{2}\right),\left(\gamma_{1}^{\prime}, \gamma_{2}^{\prime}\right)\right\}$ with the property that the paths $\gamma_{1} * \gamma_{2}$ and $\gamma_{1}^{\prime} * \gamma_{2}^{\prime}$ are homotopic in L.

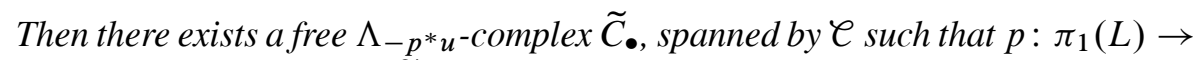
$G$ induces a morphism from $\widetilde{C}_{\bullet}$ to $C_{\bullet}$ via the natural ring morphism $p: \Lambda_{-p^{*} u} \rightarrow$ $\Lambda_{-u}$.

Proof. Fix lifts $\tilde{x} \in \tilde{L}$ of the points $x \in \mathcal{C}$. For any $h \in \pi_{1}(L)$, denote by $\tilde{\mathscr{L}}_{h}^{0}(x, y)$ the set of paths $\gamma \in \mathcal{E}$ which lift in $\tilde{L}$ to paths joining $h \tilde{x}$ and $\tilde{y}$. Then define

$$
[x, y]^{\sim}=\sum_{h \in \pi_{1}(L)} \#_{2} \tilde{\mathscr{L}}_{h}^{0}(x, y) h .
$$

Remark that $[x, y]^{\sim}$ belongs to $\Lambda_{-p^{*} u}$. Indeed it is obvious that for any $g \in G$ we have

$$
\mathscr{L}_{g}^{0}(x, y)=\bigcup_{h \in \pi_{1}(L), p(h)=g} \tilde{\mathscr{L}}_{h}^{0}(x, y) .
$$

Now define

$$
\partial x=\sum_{y \in \mathscr{C}}[x, y]^{\sim} y .
$$

Like in the case of the statement (b), the relation $\partial \circ \partial=0$ is equivalent to the fact that for any $x, z \in \mathcal{C}$ and $h \in \pi_{1}(L)$ the number of elements of the set

$$
\bigcup_{y \in \mathcal{C}, h^{\prime \prime} h^{\prime}=h} \tilde{\mathscr{L}}_{h^{\prime}}^{0}(x, y) \times \tilde{\mathscr{L}}_{h^{\prime \prime}}^{0}(y, z)
$$

is even. Let $\left(\gamma_{1}, \gamma_{2}\right)$ be an element of this set. Then the set equality above implies that

$$
\left(\gamma_{1}, \gamma_{2}\right) \in \bigcup_{y \in \mathcal{C}, g^{\prime \prime} g^{\prime}=g} \mathscr{L}_{g^{\prime}}^{0}(x, y) \times \mathscr{L}_{g^{\prime \prime}}^{0}(y, z),
$$

where $g=p(h), g^{\prime}=p\left(h^{\prime}\right)$ and $g^{\prime \prime}=p\left(h^{\prime \prime}\right)$. Let $\left(\gamma_{1}^{\prime}, \gamma_{2}^{\prime}\right)$ as in the hypothesis (c). Since $\gamma_{1} * \gamma_{2}$ and $\gamma_{1}^{\prime} * \gamma_{2}^{\prime}$ are homotopic, it follows that

$$
\gamma_{1}^{\prime} * \gamma_{2}^{\prime} \in \bigcup_{y \in \mathcal{C}, h^{\prime \prime} h^{\prime}=h} \mathscr{L}_{h^{\prime}}^{0}(x, y) \times \mathscr{L}_{h^{\prime \prime}}^{0}(y, z) .
$$

Therefore, using (c), the latter has an even number of elements. This implies that $\partial \circ \partial=0$ and proves the proposition.

We apply Proposition 3.25 to $\mathscr{C}=L \cap \phi_{1}(L)$ and $\mathcal{E}$ defined by the paths $\gamma(s)=v(s, 0)$, where $v$ are the holomorphic strips belonging to the zero-dimensional 
components of the trajectory spaces $\mathscr{L}(x, y)$. In order to check the hypothesis (c) of Proposition 3.25 recall that the space

$$
\bigcup_{y \in \mathcal{C}, g^{\prime \prime} g^{\prime}=g} \mathscr{L}_{g^{\prime}}^{0}(x, y) \times \mathscr{L}_{g^{\prime \prime}}^{0}(y, z),
$$

is the boundary of a one-dimensional closed manifold (as was shown in Lemma 3.19), so its elements can be viewed as the disjoined union of the boundaries (consisting of two elements) of the connected components of this manifold. Such a couple $\left(\gamma_{1}, \gamma_{2}\right),\left(\gamma_{1}^{\prime}, \gamma_{2}^{\prime}\right)$ has the property that $\gamma_{1} * \gamma_{2}$ and $\gamma_{1}^{\prime} * \gamma_{2}^{\prime}$ are homotopic in $L$. This

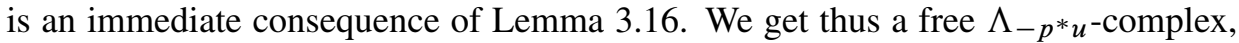
as in Proposition 3.25.

Using the equivalent approach (see Remark 3.20), we obtain a free $\Lambda_{p^{*} u}$-complex $\widetilde{C}_{\bullet}\left(L, \phi_{t}, J_{t}\right)$ spanned by the intersection points $L \cap \phi_{1}(L)$, as claimed in Theorem 3.1. In order to show that its homology only depends on $L$ and on $u$ one has to prove that the morphism of $\Lambda_{u}$-complexes

$$
\Psi_{\bullet}: C_{\bullet}\left(L, \phi_{t}^{\alpha+d H_{t}}, J_{t}\right) \rightarrow C_{\bullet}\left(L, \phi_{t}^{\alpha+d H_{t}^{\prime}}, J_{t}^{\prime}\right)
$$

defined in the previous subsection lifts to a morphism between the corresponding

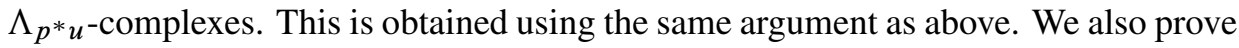
that the lifted morphism yields an isomorphism in homology in an analogous way.

The goal of this section, Theorem 3.1, is now achieved.

\section{Floer homology and Novikov homology}

Denote by $\operatorname{FH}(L, u)$ the homology of the Floer complex $\widetilde{C}_{\bullet}\left(L, \phi_{t}, J_{t}\right)$ defined in Section 3.7. Denote by $H\left(L, p^{*} u\right)$ the Novikov homology of $L$ and of the class $p^{*} u$, where $p: L \rightarrow M$ is the projection on the base space of $T^{*} M$. The aim of this section is to show that

Theorem 4.1. $\mathrm{FH}(L, u)$ is isomorphic to the Novikov homology $H\left(L, p^{*} u\right)$.

Theorem 1.1 and Corollary 1.2 will be inferred from this theorem.

4.1. Proof of Theorem 4.1. Again, we follow the ideas of [14]. We prove

Proposition 4.2. Let $u \in H^{1}(M)$ There exists an $\epsilon>0$ (depending on $u$ ) such that:

(a) For all $|\sigma|<\epsilon$,

$$
\operatorname{FH}(L,(1+\sigma) u) \approx \operatorname{FH}(L, u) .
$$


(b) $\mathrm{FH}(L, \sigma u) \approx H\left(L, p^{*} u\right)$.

Proposition 4.2 immediately implies Theorem 4.1 since the set

$$
E=\{\sigma \in] 0,+\infty\left[\mid \mathrm{FH}(L, \sigma u) \approx H_{*}\left(L, p^{*} u\right)\right\}
$$

is non empty, open and with open complementary, so it equals $] 0,+\infty[$.

Proof of Proposition 4.2. Consider the $\Lambda_{u}$-complex $C_{\bullet}\left(L, \phi_{t}, J_{t}\right)$ defined in Section 3.5 (using Remark 3.20). We showed in Section 3.6 that its homology only depends on $L$ and on $u$. Denote this homology by $\overline{\mathrm{FH}}(L, u)$. Also consider the Novikov homology associated to $p^{*} u$ and to the covering $\bar{L} \rightarrow L$ (this covering was defined in Section 3.1 as the pull-back to $L$ of the covering $T^{*} \tilde{M} \rightarrow T^{*} M$; it corresponds to $\left.\operatorname{Ker}(p) \subset \pi_{1}(L)\right)$. This homology, defined as explained in Remark 2.5, will be denoted by $H\left(\bar{L} \rightarrow L, p^{*} u\right)$. In order to show Proposition 4.2 we prove first the analogous result for the Floer homology $\overline{\mathrm{FH}}(L, u)$, namely:

Proposition 4.3. Let $u \in H^{1}(M)$ There exists an $\epsilon>0$ (depending on $u$ ) such that:

(a) For all $|\sigma|<\epsilon$,

$$
\overline{\mathrm{FH}}(L,(1+\sigma) u) \approx \overline{\mathrm{FH}}(L, u) .
$$

(b) $\overline{\mathrm{FH}}(L, \sigma u) \approx H\left(\bar{L} \rightarrow L, p^{*} u\right)$

Then, using the same method as in Section 3.7, we show that the isomorphisms in Proposition 4.3 (a) and (b) can be lifted to the isomorphisms in Proposition 4.2 (a) and (b), respectively, proving thus Proposition 4.2.

Proof of Proposition 4.3. Let $\alpha \in u$ a closed 1-form. In order to compute $\overline{\mathrm{FH}}(L, u)$ we choose a symplectic isotopy $\phi_{t}^{\alpha+d H_{t}}$, as follows. Let $\psi_{t}: T^{*} L \rightarrow T^{*} L$ the symplectic isotopy defined by $\psi_{t}(x)=x+t p^{*} \alpha$. Now we use the following wellknown result

Lemma 4.4. If $L \subset T^{*} M$ is exact Lagrangian there exists a (non-proper) symplectic embedding $\Phi: T^{*} L \rightarrow T^{*} M$, extending the given embedding of $L$. In particular, $\Phi^{*} \lambda_{M}-\lambda_{L}$ is an exact one form $d G$.

Proof. By Weinstein's theorem there is a symplectic embedding $\Phi$ of a tubular neighbourhood $U$ of $0_{L}$ whose restriction to $0_{L}$ is the given embedding of $L$. Since $L$ is exact the difference $\lambda_{M}-\left(\Phi^{-1}\right)^{*} \lambda_{L}$ is an exact 1 -form on $\Phi(U)$. This enables one to extend $\left(\Phi^{-1}\right)^{*} \lambda_{L}$ to a primitive of $\omega_{M}$ on $T^{*} M$. The symplectic dual of this primitive is a vector field whose restriction on $\Phi(U)$ is the image of the canonical Liouville vector field on $U \subset T^{*} L$. Denote by $\xi_{t}$ the flow of this vector field and by 
$\rho_{t}$ the flow of the canonical Liouville vector field on $T^{*} L$. Then the embedding $\Phi$ is defined by the formula

$$
\Phi(x)=\left.\xi_{t} \circ \Phi\right|_{U} \circ \rho_{-t}(x),
$$

where $t>0$ is sufficiently large to ensure $\rho_{-t}(x) \in U$. It is easy to see that this definition does not depend on $t$ and that $\Phi$ is an exact symplectic embedding as claimed.

Consider now the Lagrangian isotopy $\left.\Phi \circ \psi_{t}\right|_{L}: L \rightarrow T^{*} M$. There is a symplectic isotopy $\left(\phi_{t}\right)$ on $T^{*} M$ which extends $\Phi \circ \psi_{t}$. To see this, one has to consider the isotopy $\chi_{t}: T^{*} M \rightarrow T^{*} M$ defined by $\chi_{t}(x)=x+t \alpha$. It is easy to see that $\left(\chi_{-t} \psi_{t}\right)^{*} \lambda_{M}$ is an exact 1-form on $L$, so $\chi_{-t} \psi_{t}$ is an exact Lagrangian isotopy. Consider a Hamiltonian extension $\left(\Gamma_{t}\right)$ of $\chi_{-t} \psi_{t}$. Then $\chi_{t} \Gamma_{t}$ is an extension of $\psi_{t}$. Therefore we can consider a symplectic extension of $\Phi \circ \psi_{t}$, which we denote by $\left(\phi_{t}\right)$. The Calabi invariant of the extension is clearly $u=[\alpha]=\operatorname{Cal}\left(\psi_{t}\right)$ since $\left(\Gamma_{t}\right)$ is Hamiltonian and $\Phi$ is an extension of an exact Lagrangian embedding.

Using Lemma 3.3, we may suppose that $\left(\phi_{t}\right)$ is defined by $\alpha+d H_{t}$, where $H:[0,1] \times T^{*} M \rightarrow \mathbb{R}$ is compactly supported. We will use this isotopy for the definition of the Floer complex. Note that the intersection points $L \cap \phi_{t}(L)$ are the zeroes of $p^{*} \alpha$ in $L$ and therefore they are fixed with respect to $t$. In other words, the zeroes of the action 1-form $\hat{v}$ are constant paths in $\Omega(L, L)$. Note also that when $\alpha$ is Morse (which we will always suppose) the intersections $L \cap \phi_{t}(L)$ are transverse, so the isotopy $\left(\phi_{t}\right)$ is generic in this sense.

We will also need the following Palais-Smale-type lemma (see [14], Lemma 5.1)

Lemma 4.5. Let $L_{t}=\phi_{t}(L)$, as above and denote by $\left\{x_{1}, \ldots, x_{k}\right\}$ the intersection points $L \cap L_{t}$ for $t>0$. Fix a ball $B_{i} \subset T^{*} M$ around each $x_{i}$ and denote by $B$ the union $\bigcup_{i} B_{i}$. Then there exist $c>0$ such that for any smooth $z \in \Omega(L, L)$, whose image is not contained in $B$ we have

$$
\left\|z^{\prime}(t)-X^{\alpha+d H_{t}}(z(t))\right\|_{L^{2}} \geq c .
$$

Proof. The norm $L^{2}$ in the statement above is defined using a fixed complete metric on $T^{*} M$. Suppose the contrary of the assertion: there exist a sequence $\left(z_{n}\right) \in \Omega(L, L)$ of paths whose images are not contained in $B$, such that

$$
\lim _{n \rightarrow+\infty}\left\|z_{n}^{\prime}(t)-X^{\alpha+d H_{t}}\left(z_{n}(t)\right)\right\|_{L^{2}}=0 .
$$

As $H_{t}$ is compactly supported and $\alpha$ is defined on $M$ the norm $\left\|X^{\alpha}(z)+X^{d H_{t}}(z)\right\|_{L^{2}}$ is bounded uniformly with respect to $z$, so there is a constant $K>0$ such $\left\|z_{n}^{\prime}\right\|_{L^{2}} \leq K$ for all $n \in \mathbb{N}$. Let $d$ be the distance defined on $T^{*} M$ by the metric we considered. 
For arbitrary $t_{0}<t_{1}$ in $[0,1]$ we have

$$
\begin{aligned}
d\left(z_{n}\left(t_{0}\right), z_{n}\left(t_{1}\right)\right) & \leq \int_{t_{0}}^{t_{1}}\left\|z_{n}^{\prime}(t)\right\| d t=\int_{0}^{1} 1_{\left[t_{0}, t_{1}\right]}\left\|z_{n}^{\prime}(t)\right\| d t \\
& \leq\left\|z_{n}^{\prime}\right\|_{L^{2}}\left\|1_{\left[t_{0}, t_{1}\right]}\right\|_{L^{2}} \leq K \sqrt{t_{1}-t_{0}} .
\end{aligned}
$$

The family $\left(z_{n}\right)$ is therefore equicontinuous. Since $\left(z_{n}(0)\right) \in L$ admits a convergent subsequence, we may apply Arzela-Ascoli to get a subsequence of $\left(z_{n}\right)$ which converges towards some $z_{\infty} \in \Omega(L, L)$ in the topology $\ell^{0}\left([0,1], T^{*} M\right)$. It follows that $X^{\alpha+d H_{t}}\left(\left(z_{n}\right)\right.$ converges towards $X^{\alpha+d H_{t}}\left(z_{\infty}\right)$ in the topology $\ell^{0}$ and in particular in the norm $L^{2}$.

But $\left\|z_{n}^{\prime}(t)-X^{\alpha+d H_{t}}\left(z_{n}(t)\right)\right\|_{L^{2}}$ converges to zero, so we have

$$
\lim _{n \rightarrow+\infty}\left\|z_{n}^{\prime}(t)\right\|_{L^{2}}=\left\|X^{\alpha+d H_{t}}\left(z_{\infty}(t)\right)\right\| .
$$

Embed $T^{*} M$ is some Euclidean space $\mathbb{R}^{N}$ and see the vectors fields in the equality above as elements of $\ell^{0}\left([0,1], \mathbb{R}^{N}\right)$ (depending on the variable $t$ ). Obviously the last convergence is valid in $L^{2}\left([0,1], \mathbb{R}^{N}\right)$. Then one can write for $t \in[0,1]$

$$
\begin{aligned}
z_{n}(t)-z_{n}(0) & =\int_{0}^{t} z_{n}^{\prime}(\tau) d \tau \\
& =\int_{0}^{t} z_{n}^{\prime}(\tau)-X^{\alpha+d H_{t}}\left(z_{\infty}(\tau)\right) d \tau+\int_{0}^{t} X^{\alpha+d H_{t}}\left(z_{\infty}(\tau)\right) d \tau .
\end{aligned}
$$

Using the Cauchy-Schwarz inequality, we find as above

$$
\left\|\int_{0}^{t} z_{n}^{\prime}(\tau)-X^{\alpha+d H_{t}}\left(z_{\infty}(\tau)\right) d \tau\right\| \leq\left\|z_{n}^{\prime}-X^{\alpha+d H_{t}}\left(z_{\infty}\right)\right\|_{L^{2}} \sqrt{t},
$$

in particular this integral converges to zero. We infer that when $n$ goes to $+\infty$ the preceding equality can be written as

$$
z_{\infty}(t)-z_{\infty}(0)=\int_{0}^{t} X^{\alpha+d H_{t}}\left(z_{\infty}(\tau)\right) d \tau .
$$

In particular, $z_{\infty}$ is $\mathcal{C}^{1}$ (and hence $\mathcal{C}^{\infty}$, by an obvious bootstrapping argument) and satisfies

$$
z_{\infty}^{\prime}=X^{\alpha+d H_{t}}\left(z_{\infty}\right)
$$

This means that $z_{\infty}$ is a zero of the action 1-form $\hat{v}$, hence it is a constant path which belongs to $\left\{x_{1}, \ldots, x_{k}\right\}$. But this is contradictory, since the image of $z_{n}$ is not contained in $B$, so the sequence $\left(z_{n}\right)$ cannot converge towards an element $z_{\infty} \in\left\{x_{1}, \ldots, x_{k}\right\}$. 
Now we are able to give the

Proof of Proposition 4.3 (a). Recall that we have a generic isotopy $\left(\phi_{t}\right)$ which is defined by $X^{\alpha+d H_{t}}$. The intersection points $L \cap \phi_{t}(L)$ are fixed with respect to $t$; we denoted them $\left\{x_{1}, \ldots, x_{k}\right\}$. As in Lemma 4.5 we fix a collection of balls around these intersection points and we denote its union by $B$. We also consider the constant $c$ given by this lemma. Recall also that $u \in H^{1}(M)$ is the cohomology class of $\alpha$.

Choose $\eta \in u$ such that $\left.\eta\right|_{B}=0$ and fix $\epsilon>0$ such that

$$
\epsilon\|\eta\|<c / 3
$$

Then pick $\sigma<\epsilon$ and consider the symplectic isotopy $\left(\psi_{t}\right)$ defined by $X^{\alpha+\sigma \eta+d H_{t}}$. The constant $\epsilon>0$ is chosen small enough to ensure that $\psi_{1}(L)$ is still transverse to $L$ (actually we may even suppose that the intersection points are $\left\{x_{1}, \ldots, x_{k}\right\}$ but this is not needed in the proof). Now fix a compatible almost complex structure $J$ on $T^{*} M$ which yields a complete metric. Then choose compatible complex structures $J_{t}$ and $J_{t}^{\prime}$ such that the couples $\left(\alpha+d H_{t}, J_{t}\right)$ and $\left(\alpha+d H_{t}+\sigma \eta, J_{t}^{\prime}\right)$ satisfy the transversality assumption of Theorem 3.12. By genericity, we may suppose that $\left\|J_{t}-J\right\|<\delta$ and $\left\|J_{t}^{\prime}-J\right\|<\delta$ where $\delta>0$ is a (small) constant which will be specified later. The norm here is defined by the metric $g_{J}$, induced by $J$. Like in the previous section, define the $\Lambda_{u}$-complexes $C_{\bullet}\left(L, \phi_{t}^{\alpha+d H_{t}}, J_{t}\right)$ and $C_{\bullet}\left(L, \psi_{t}^{\alpha+\sigma \eta+d H_{t}}, J_{t}^{\prime}\right)$ (we use here that $\Lambda_{u}=\Lambda_{\tau u}$ for any $\left.\tau>0\right)$.

To finish the proof of Proposition 4.3 (a) we have to prove that the homologies of these two complexes are isomorphic. We proceed as in Section 3.6 by constructing a homotopy between the pairs $\left(\alpha+d H_{t}, J_{t}\right)$ and $\left(\alpha+\sigma \eta+d H_{t}, J_{t}^{\prime}\right)$. Denote by $\left(\alpha+\chi(s) \sigma \eta+d H_{t}, J_{t, s}\right)$ this homotopy. Here $\chi$ is a monotone increasing smooth function on $\mathbb{R}$ which vanishes for $s \leq-R$ and equals 1 for $s \geq R$. We chose the homotopy of almost complex structures such that: $J_{s, t}=J_{t}$ for $s \leq-R$ and $J_{s, t}=J_{t}^{\prime}$ for $s \geq R$. We may also suppose that for all $s \in \mathbb{R}\left\|J_{s, t}-\bar{J}\right\|<\delta$. In order to define a morphism between the two complexes above we need to consider the solutions $v: \mathbb{R} \times[0,1] \rightarrow T^{*} M$ of the system

$$
\left\{\begin{array}{l}
\frac{\partial v}{\partial s}+J_{s, t}\left(\frac{\partial v}{\partial t}-X^{\alpha+d H_{t}+\chi(s) \sigma \eta}(v)\right)=0 \\
v(s, i) \in L \text { for } i=0,1 \\
E(v)<+\infty
\end{array}\right.
$$

where $E(v)$ is the energy of $v$ with respect to the norm defined by $J$ (or equivalently, to the norm defined by $\left.J_{s, t}\right)$. As in the previous section, each solution $v$ of this system satisfies $\lim _{s \rightarrow-\infty} v(s, t)=x(t)$ and $\lim _{s \rightarrow+\infty} v(s, t)=y(t)$, where $x(t) \in$ $\Omega(L, L)$ is an orbit of $X^{\alpha+d H_{t}}$ and $y(t) \in \Omega(L, L)$ is an orbit of $X^{\alpha+\sigma \eta+d H_{t}}$ (equivalently, they are zeroes of the corresponding action 1-forms). The genericity assumptions ensure that the spaces $\mathcal{M}_{\chi, J_{s, t}}(x, y)$ of solutions with the indicated limit 
conditions are manifolds of local dimension $\mu(v)$. As in Theorem 3.15, the zero dimensional subspaces $\mathcal{M}_{\chi, J_{s, t}}^{0}(x, y ; A)$ of solutions with energy uniformly bounded by $A$ are compact and those of dimension 1 are compact up to breaking into $\left(v^{1}, v^{2}\right)$ where only one of the $v^{i}$ 's is a solution of the equation above, the other being a solution of the Floer equation corresponding either to $\left(\alpha+d H_{t}, J_{t}\right)$ or to $\left(\alpha+\sigma \eta+d H_{t}, J_{t}^{\prime}\right)$. We want to define a morphism

$$
\Gamma_{\bullet}: C_{\bullet}\left(L, \phi_{t}^{\alpha+d H_{t}}, J_{t}\right) \rightarrow C_{\bullet}\left(L, \psi_{t}^{\alpha+\sigma \eta+d H_{t}}, J_{t}^{\prime}\right)
$$

by the formula

$$
\Gamma(x)=\sum_{g \in \pi_{1}(M), y} m_{g}(x, y) g y,
$$

where $m_{g}(x, y)$ is the number (modulo 2) of elements of the space $\mathcal{M}_{g, s}^{0}(x, y) \subset$ $\mathcal{M}_{\chi, J_{s, t}}^{0}(x, y)$ of solutions which lift to $T^{*} \tilde{M}$ starting form $g \bar{x}$ and ending at $\bar{y}$ (as previously, we fixed lifts $\bar{x}$ and $\bar{y}$ for all the zeroes of the two action 1-forms).

The crucial point is the following statement, analogous to Proposition 3.23. It implies that $\mathcal{M}_{g, s}^{0}(x, y)$ is finite and that for any $y$ the sum $\sum_{g \in \pi_{1}(M)} m_{g}(x, y) g$ belongs to $\Lambda_{u}$ :

Proposition 4.6. The space $\mathcal{M}_{g, s}(x, y)$ is contained in $\mathcal{M}_{\chi, J_{s, t}}(x, y ; A)$ for some $A>0$.

Proof. Let $v \in \mathcal{M}_{g, s}(x, y)$. As in the previous section $\hat{\mathcal{A}}$ and $\hat{\mathcal{A}}^{\prime}$ are the primitives of the pull-backs to $T^{*} \widetilde{M}$ of the two action 1-forms $\hat{v}$ and $\hat{v}^{\prime}$. Denote by $\bar{v}$ the lift of $v$ to $T^{*} \tilde{M}$. The proof of the proposition is implied by the estimate

$$
E(v) \leq 3[\hat{\mathcal{A}}(\bar{x})-\hat{\mathcal{A}}(\bar{y})+u(g)] .
$$

Let us prove this inequality. In the estimations below the scalar product $\langle$,$\rangle is$ $g_{J}(\cdot, \cdot)=\omega_{M}(\cdot, J \cdot)$ for the fixed structure $J$ and the norm $\|\cdot\|$ is defined by this metric. We have

$$
\begin{aligned}
\hat{\mathcal{A}}(g \bar{x})-\hat{\mathcal{A}}(\bar{y}) & =-\int_{-\infty}^{+\infty} \frac{\partial}{\partial s} \hat{\mathcal{A}}(\bar{v}(s, \cdot)) d s \\
& =-\int_{-\infty}^{+\infty} \hat{v}\left(\frac{\partial v}{\partial s}\right) d s=-\int_{-\infty}^{+\infty}\left\langle\frac{\partial v}{\partial s}, \operatorname{grad}_{v}^{g_{J}} \hat{v}\right\rangle d s \\
& =-\int_{-\infty}^{+\infty} \int_{0}^{1}\left\langle\frac{\partial v}{\partial s}, J\left(\frac{\partial v}{\partial t}-X^{\alpha+d H_{t}}(v)\right)\right\rangle d t d s .
\end{aligned}
$$

In order to prove (6) we have to find a lower bound for the right term of the previous equality. Let us fix $s \in \mathbb{R}$. Recall that $B$ is a fixed union of balls around 
the intersection points $L \cap \phi_{t}(L)$ such that Lemma 4.5 is valid. We consider the following cases:

Case 1. $\operatorname{Im}(v(s, \cdot)) \subset B$. Using that $v$ is a solution of the parametrized Floer equation, we get

$$
\begin{aligned}
\int_{0}^{1}\left\langle\frac{\partial v}{\partial s}, J\left(\frac{\partial v}{\partial t}-X^{\alpha+d H_{t}}(v)\right)\right\rangle d t \\
\quad=\int_{0}^{1}\left\langle\frac{\partial v}{\partial s}, J\left(J_{t, s} \frac{\partial v}{\partial s}+X^{\alpha+\chi(s) \sigma \eta+d H_{t}}(v)-X^{\alpha+d H_{t}}(v)\right\rangle d t\right. \\
\quad=\int_{0}^{1}\left\langle\frac{\partial v}{\partial s}, J J_{t, s} \frac{\partial v}{\partial s}\right\rangle d t
\end{aligned}
$$

since $\left.\eta\right|_{B}=0$ (so $\left.X^{\chi(s) \sigma \eta}(v)=0\right)$. It follows that

$$
\begin{aligned}
\int_{0}^{1}\left\langle\frac{\partial v}{\partial s}, J\left(\frac{\partial v}{\partial t}-X^{\alpha+d H_{t}}(v)\right)\right\rangle d t \\
\quad=-\int_{0}^{1}\left\langle J \frac{\partial v}{\partial s}, J_{s, t} \frac{\partial v}{\partial s}\right\rangle d t=-\int_{0}^{1}\left\|\frac{\partial v}{\partial s}\right\|^{2} d t+\int_{0}^{1}\left\langle J \frac{\partial v}{\partial s},\left(J_{s, t}-J\right) \frac{\partial v}{\partial s}\right\rangle d t \\
\quad \leq-\int_{0}^{1}\left\|\frac{\partial v}{\partial s}\right\|^{2} d t+\delta \int_{0}^{1}\left\|\frac{\partial v}{\partial s}\right\|^{2} d t=-(1-\delta)\left\|\frac{\partial v}{\partial s}\right\|_{L^{2}}^{2}
\end{aligned}
$$

Choosing $\delta<2 / 3$ we get

$$
\int_{0}^{1}\left\langle\frac{\partial v}{\partial s}, J\left(\frac{\partial v}{\partial t}-X^{\alpha+d H_{t}}(v)\right)\right\rangle d t \leq-1 / 3\left\|\frac{\partial v}{\partial s}\right\|_{L^{2}}^{2} .
$$

Case 2. $\operatorname{Im}(v(s, \cdot)) \not \subset B$. Proceeding as above we obtain

$$
\begin{aligned}
\int_{0}^{1}\left\langle\frac{\partial v}{\partial s}, J\left(\frac{\partial v}{\partial t}-X^{\alpha+d H_{t}}(v)\right)\right\rangle d t \\
\quad=\int_{0}^{1}\left\langle\frac{\partial v}{\partial s}, J\left(J_{t, s} \frac{\partial v}{\partial s}+X^{\alpha+\chi(s) \sigma \eta+d H_{t}}(v)-X^{\alpha+d H_{t}}(v)\right)\right\rangle d t \\
\quad=\int_{0}^{1}\left\langle\frac{\partial v}{\partial s}, J J_{t, s} \frac{\partial v}{\partial s}\right\rangle d t+\int_{0}^{1}\left\langle\frac{\partial v}{\partial s}, J X^{\chi(s) \sigma \eta}(v)\right\rangle d t \\
=-\int_{0}^{1}\left\langle J \frac{\partial v}{\partial s}, J_{s, t} \frac{\partial v}{\partial s}\right\rangle d t-\int_{0}^{1} \omega_{M}\left(\frac{\partial v}{\partial s}, X^{\chi(s) \sigma \eta}(v)\right) d t \\
=-\int_{0}^{1}\left\|\frac{\partial v}{\partial s}\right\|^{2} d t+\int_{0}^{1}\left\langle J \frac{\partial v}{\partial s},\left(J_{s, t}-J\right) \frac{\partial v}{\partial s}\right\rangle d t-\int_{0}^{1} \chi(s) \sigma \eta\left(\frac{\partial v}{\partial s}\right) d t \\
\leq-(1-\delta)\left\|\frac{\partial v}{\partial s}\right\|_{L^{2}}^{2}+\epsilon\|\eta\| \int_{0}^{1}\left\|\frac{\partial v}{\partial s}\right\| d t
\end{aligned}
$$


We apply the Cauchy-Schwarz inequality to the last integral and the fact that $\epsilon$ was chosen to satisfy $\epsilon\|\eta\|<c / 3$. We have therefore

$$
\int_{0}^{1}\left\langle\frac{\partial v}{\partial s}, J\left(\frac{\partial v}{\partial t}-X^{\alpha+d H_{t}}(v)\right)\right\rangle d t \leq-(1-\delta)\left\|\frac{\partial v}{\partial s}\right\|_{L^{2}}^{2}+\frac{c}{3}\left\|\frac{\partial v}{\partial s}\right\|_{L^{2}} .
$$

We have not used the condition $\operatorname{Im}(v(s, \cdot)) \not \subset B$ yet. Note that it implies using Lemma 4.5 that

$$
\left\|\frac{\partial v}{\partial t}-X^{\alpha+d H_{t}}(v)\right\| \geq c .
$$

We infer:

$$
\begin{aligned}
& \left\|\frac{\partial v}{\partial s}\right\|_{L^{2}}=\left\|J_{s, t}\left(\frac{\partial v}{\partial t}-X^{\alpha+\chi(s) \sigma \eta+d H_{t}}\right)\right\|_{L^{2}} \\
& \quad \geq\left\|J\left(\frac{\partial v}{\partial t}-X^{\alpha+\chi(s) \sigma \eta+d H_{t}}\right)\right\|_{L^{2}}-\left\|\left(J-J_{s, t}\right)\left(\frac{\partial v}{\partial t}-X^{\alpha+\chi(s) \sigma \eta+d H_{t}}\right)\right\|_{L^{2}} \\
& \quad \geq(1-\delta)\left\|\frac{\partial v}{\partial t}-X^{\alpha+\chi(s) \sigma \eta+d H_{t}}\right\|_{L^{2}} \\
& \quad \geq(1-\delta)\left(\left\|\frac{\partial v}{\partial t}-X^{\alpha+d H_{t}}\right\|_{L^{2}}-\left\|X^{\chi(s) \sigma \eta}\right\|_{L^{2}}\right) \\
& \quad \geq(1-\delta)\left(\left\|\frac{\partial v}{\partial t}-X^{\alpha+d H_{t}}\right\|_{L^{2}}-\epsilon\|\eta\|\right) \\
& \quad \geq(1-\delta)(c-c / 3)=\frac{2}{3}(1-\delta) c,
\end{aligned}
$$

using Lemma 4.5. Let us come back to the inequality (9). Using (10) we find that for $\delta$ small enough we have

$$
-(1-\delta)\left\|\frac{\partial v}{\partial s}\right\|_{L^{2}}^{2}+\frac{c}{3}\left\|\frac{\partial v}{\partial s}\right\|_{L^{2}} \leq-1 / 3\left\|\frac{\partial v}{\partial s}\right\|_{L^{2}}^{2} .
$$

Indeed, this is equivalent to

$$
\left(\frac{2}{3}-\delta\right)\left\|\frac{\partial v}{\partial s}\right\|_{L^{2}} \geq c / 3
$$

which, using (10), is implied by

$$
2(1-\delta)\left(\frac{2}{3}-\delta\right) \geq 1,
$$

and this is true if we take for instance $\delta<1 / 10$.

This means that in the case 2 we also have

$$
\int_{0}^{1}\left\langle\frac{\partial v}{\partial s}, J\left(\frac{\partial v}{\partial t}-X^{\alpha+d H_{t}}(v)\right)\right\rangle d t \leq-1 / 3\left\|\frac{\partial v}{\partial s}\right\|_{L^{2}}^{2},
$$


and (8) and (11) imply that the above inequality is valid for any $s \in \mathbb{R}$. Now we integrate this inequality with respect to $s$ and we get using (7) that

$$
\hat{\mathcal{A}}(g \bar{x})-\hat{\mathcal{A}}(\bar{y}) \geq 1 / 3 \int_{-\infty}^{+\infty}\left\|\frac{\partial v}{\partial s}\right\|_{L^{2}[0,1]}^{2} d s=\frac{1}{3} E(v) .
$$

This implies the inequality (6) and finishes the proof.

Therefore the space $\mathcal{M}_{g, s}^{0}(x, y)$ is finite. Moreover, if $m_{g}(x, y)$ is its parity, the inequality (6) in the proof of Proposition 4.6 shows that the sum

$$
\sum_{g \in \pi_{1}(M)} m_{g}(x, y) g
$$

belongs to $\Lambda_{u}$. This enables us to define the morphism

$$
\Gamma_{\bullet}: C_{\bullet}\left(L, \phi_{t}^{\alpha+d H_{t}}, J_{t}\right) \rightarrow C_{\bullet}\left(L, \psi_{t}^{\alpha+\sigma \eta+d H_{t}}, J_{t}^{\prime}\right) .
$$

The fact that $\Gamma_{\bullet}$ commutes with the differentials is proved by studying the compactness of the 1-dimensional components $\mathcal{M}_{g, s}^{1}(x, y)$. The proof is analogous to that of Lemma 3.19. To show that $\Gamma$. induces an isomorphism at the level of homology, one should proceed as usual: Take a homotopy between $\left(\alpha+\sigma \eta+d H_{t}, J_{t}^{\prime}\right)$ and $\left(\alpha+d H_{t}, J_{t}\right)$. This yields a morphism

$$
\Gamma_{\bullet}^{\prime}: C_{\bullet}\left(L, \psi_{t}^{\alpha+\sigma \eta+d H_{t}}, J_{t}^{\prime}\right) \rightarrow C_{\bullet}\left(L, \phi_{t}^{\alpha+d H_{t}}, J_{t}\right) .
$$

Then, using two-parameter homotopies as in [11] one can show that $\Gamma_{\bullet} \Gamma_{\bullet}^{\prime}$ is homotopic to the identity and also that $\Gamma_{\bullet}^{\prime} \Gamma_{\bullet}$ is homotopic to the identity. This shows that the homologies of the complexes are isomorphic, and the proof of Proposition 4.3 (a) is finished.

Proof of Proposition 4.3 (b). Replace overall in the proof of Proposition 4.3 (a) the 1 -form $\alpha \in u$ with an exact 1 -form $d f$ where $f: M \rightarrow \mathbb{R}$. We get then in a similar manner a $\Lambda_{u}$-morphism

$$
\Gamma_{\bullet}^{0}: C_{\bullet}\left(L, \phi_{t}^{d f+d H_{t}}, J_{t}\right) \rightarrow C_{\bullet}\left(L, \phi_{t}^{d f+\sigma \eta+d H_{t}}, J_{t}^{\prime}\right),
$$

which is an isomorphism in homology. The first complex above is actually a $\Lambda$-complex with coefficients extended to $\Lambda_{u}$. Replace it by $\Lambda_{u} \otimes_{\Lambda} C_{\bullet}\left(L, \phi_{t}^{d f+d H_{t}}, J_{t}\right)$. Now the Hamiltonian invariance implies that the homotopy class of the $\Lambda$-complex $C_{\bullet}\left(L, \phi_{t}^{d f+d H_{t}}, J_{t}\right)$ does not depend on the choice of a regular pair $\left(d f+d H_{t}, J_{t}\right)$. On the other hand, the classical Floer argument [7] provides a regular pair $\left(H_{t}^{0}, J_{t}^{0}\right)$ such that the complex $C_{\bullet}\left(L, \phi_{t}^{d H_{t}^{0}}, J_{t}^{0}\right)$ coincides with the Morse complex defined 
by a pair $(g, \xi)$ - where $g: L \rightarrow \mathbb{R}$ is a Morse function and $\xi$ is a generic pseudogradient on $L-$ in the following sense: the complex $C_{\bullet}\left(\phi_{t}^{d H_{t}^{0}}, J_{t}^{0}\right)$ is spanned by the intersection points $L \cap(L+d g)$ in $T^{*} L \subset T^{*} M$ and the map

$$
v(s, t) \mapsto v(s, 0)
$$

is a one-to-one correspondence between the holomorphic strips which define the Floer differential and the trajectories of $\xi$ which define the Morse differential.

It is then easy to see that the above Morse complex is identical to $C .(\bar{L} \rightarrow L, \xi)$, where $\bar{L} \rightarrow L$ is the pull-back of $T^{*} \widetilde{M} \rightarrow T^{*} M$ : this is the Morse complex defined using $(g, \xi)$, by lifting the trajectories of $\xi$ to $\bar{L}$, as in Section 2.2 (see Remark 2.5). Moreover, the Novikov ring which defines the Novikov homology associated to the class $p^{*} u$ and to the covering $\bar{L} \rightarrow L$ is the same as $\Lambda_{u}$. Finally, if we denote by $\sim$ the relation of homotopy equivalence between $\Lambda_{u}$-complexes we get

$$
\Lambda_{u} \otimes_{\Lambda} C_{\bullet}\left(L, \phi_{t}^{d f+d H_{t}}, J_{t}\right) \sim \Lambda_{u} \otimes_{\Lambda} C_{\bullet}\left(L, \phi_{t}^{d H_{t}^{0}}, J_{t}^{0}\right) \sim \Lambda_{u} \otimes_{\Lambda} C_{\bullet}(\bar{L} \rightarrow L, g, \xi) .
$$

The latter complex defines the Novikov homology $H\left(\bar{L} \rightarrow L, p^{*} u\right)$ corresponding to the covering $\bar{L} \rightarrow L$. We therefore have the isomorphism

$$
\overline{\mathrm{FH}}(L, \sigma u) \approx H\left(\bar{L} \rightarrow L, p^{*} u\right),
$$

and the proof of Proposition 4.3 (b) is complete.

End of the proof of Proposition 4.2. We argue as in Section 3.7. The holomorphic strips of the moduli space $\mathcal{M}_{g, s}^{0}(x, y)$ define paths on $L$ from $x$ to $y$ by the formula:

$$
v \mapsto v(s, 0) .
$$

For fixed lifts $\tilde{x}, \tilde{y} \in \tilde{L}$, such a path lifts to $\tilde{L}$ to a path joining $h \tilde{x}$ and $\tilde{y}$ for some $h \in \pi_{1}(L)$. We define thus the spaces $\widetilde{\mathcal{M}}_{h, s}^{0}(x, y)$. Obviously, we have

$$
\mathcal{M}_{g, s}^{0}(x, y)=\bigcup_{\substack{h \in \pi_{1}(L) \\ p(h)=g}} \tilde{\mathcal{M}}_{h, s}^{0}(x, y) .
$$

In particular, any set $\widetilde{\mathcal{M}}_{h, s}^{0}(x, y)$ has a finite number of elements (by Proposition 4.6) and the formula

$$
\widetilde{\Gamma}(x)=\sum_{h \in \pi_{1}(L), y} m_{h}(x, y) h y
$$

- where $m_{h}(x, y)$ is the number modulo 2 of elements in $\tilde{\mathcal{M}}_{h, s}^{0}(x, y)$ - defines a morphism of $\Lambda_{p^{*} u \text {-complexes }}$

$$
\widetilde{\Gamma}_{\bullet}: \widetilde{C}_{\bullet}\left(L, \phi_{t}^{\alpha+d H_{t}}, J_{t}\right) \rightarrow \widetilde{C}_{\bullet}\left(L, \psi_{t}^{\alpha+\sigma \eta+d H_{t}}, J_{t}^{\prime}\right)
$$


which is a lift of the morphism $\Gamma$. defined in the proof of Proposition 4.3. Using the same argument we show that it induces an isomorphism in homology, proving thus (a). Then, we proceed analogously in order to define a lift of the morphism $\Gamma_{\bullet}^{0}$ of the proof of Proposition 4.3 (b). For the particular choice $\left(H_{t}^{0}, J_{t}^{0}\right)$ in Proposition $4.3(\mathrm{~b})$, the lifted complex $\widetilde{C} \bullet$ is the Morse complex defined on the universal cover $\tilde{L}$. Therefore, the same argument as in the proof of Proposition 4.3 (b) yields an isomorphism

$$
H F(L, \sigma u) \approx H\left(L, p^{*} u\right),
$$

completing the proof of Proposition 4.2.

As we explained, this immediately implies Theorem 4.1 .

\subsection{Proof of the main results}

Proof of Theorem 1.3. If $M$ is the total space of a fibration over the circle and $L \subset$ $T^{*} M$ is exact, we consider a non-vanishing closed 1-form $\alpha$ on $M$ and we define the symplectic isotopy $\Psi_{t}(p, q)=\left(p+t \alpha_{q}, q\right)$. Obviously $\Psi_{T}(L) \cap L=\emptyset$ for $T$ sufficiently large. Without restricting the generality we may suppose that $T=1$. The Floer complex defined in the previous subsection is empty so $\mathrm{FH}(L, u)=0$, where $u$ is the cohomology class of $\alpha$. Using Theorem 4.1 this implies Theorem 1.3, i.e.,

$$
H_{*}\left(L, p^{*} u\right)=0 .
$$

Note that since $p: \pi_{1}(L) \rightarrow \pi_{1}(M)$ is an epimorphism the class $p^{*} u$ is not zero.

Proof of Theorem 1.1. (a) Let $<g_{1}, g_{2}, \ldots, g_{p} \mid r_{1}, \ldots, r_{q}>$ be a presentation of $\pi_{1}(L)$. If $p-q \geq 2$ then by Proposition 2.3 (a) we have that $H_{1}\left(L, p^{*} u\right) \neq 0$, contradicting (12). We infer that $p-q \leq 1$, as claimed.

(b) If $\pi_{1}(L)=G_{1} * G_{2}$ for some non trivial groups $G_{i}$, then we have again $H_{1}\left(L, p^{*} u\right) \neq 0$, by Proposition $2.3(\mathrm{~b})$, and the proof is finished.

Proof of Corollary 1.2. (a) Suppose that there exists an exact Lagrangian embedding

$$
L \times P \hookrightarrow T^{*}\left(Q \times S^{1}\right),
$$

where $\chi(L) \neq 0$ and $\pi_{1}(P)$ is finite. As above, we obtain $H_{*}\left(L \times P, p^{*} u\right)=0$, where $u$ is the class of the nonvanishing closed 1 -form $d \theta$ on $Q \times S^{1}$. Since $\pi_{1}(P)$ is finite, we obtain $p^{*} u \in H^{1}(L, \mathbb{R}) \subset H^{1}(L \times P, \mathbb{R})$. We apply Proposition 2.6 and we obtain $H_{*}\left(L, p^{*} u\right)=0$. But this contradicts Remark 2.7.

(b) Suppose that there exists a Lagrangian embedding

$$
\left(L_{1} \# L_{2}\right) \times P \hookrightarrow T^{*}\left(Q \times S^{1}\right) .
$$


We show that either $L_{1}$ or $L_{2}$ is a simply connected $\mathbb{Z} / 2$-homology sphere. As $n \geq 4$, the fundamental group of $L=L_{1} \# L_{2}$ is the free product $\pi_{1}\left(L_{1}\right) * \pi_{1}\left(L_{2}\right)$. We get from Theorem 1.1 that one of the $L_{i}$ 's is simply connected. Suppose $\pi_{1}\left(L_{1}\right)=1$.

By Theorem 1.3 we know that $H_{*}\left(L, p^{*} u\right)=0$, where $u$ is the class $d \theta$ on $M=Q \times S^{1}$ and $p$ is the projection. We show that this Novikov homology cannot vanish unless $L_{1}$ is a simply connected $\mathbb{Z} / 2$-homology sphere.

Denote $D_{i} \subset L_{i}$ two embedded open $n$-disks and write $L$ as

$$
\left(L_{1} \backslash D_{1}\right) \bigcup_{S^{n-1}}\left(S^{n-1} \times[0,1]\right) \bigcup_{S^{n-1}}^{\bigcup}\left(L_{2} \backslash D_{2}\right) .
$$

Choose a CW-structure on $L$ which fits to this decomposition and which is the standard product structure on $S^{n-1} \times[0,1]$ (we take the decomposition with one 0-cell and one $(n-1)$-cell on $\left.S^{n-1}\right)$. Denote by $D \bullet$ the $\mathbb{Z} / 2$-free subcomplex spanned by the cells of $L_{1} \backslash D_{1}$. Consider the Novikov complex $C_{\bullet}\left(L, p^{*} u\right)$ which is by definition the tensor product $\Lambda_{p^{*} u} \otimes_{\mathbb{Z} / 2\left[\pi_{1}(L)\right]} C_{\bullet}(\tilde{L})$. Since $L_{1}$ is simply connected, $\tilde{L}$ is the connected sum of $\tilde{L}_{2}$ with $\pi_{1}(L)$ copies of $L_{1}$. Using this fact and our choice of the $\mathrm{CW}$-structure, we get that the complex

$$
\Lambda_{p^{*} u} \otimes_{\mathbb{Z} / 2} D_{1 \leq \bullet \leq n-1}
$$

is a direct summand in $C_{\bullet}\left(L, p^{*} u\right)$. In particular, since the Novikov complex is acyclic (by Theorem 1.3), the homology of $D_{\bullet}$, which is the homology $H_{i}\left(L_{1} \backslash D_{1} ; \mathbb{Z} / 2\right)$, vanishes in degrees $2 \leq i \leq n-2$. Then, $L_{1} \backslash D_{1}$ is simply connected so the homology $H_{1}\left(L_{1} \backslash D_{1}\right)$ vanishes, too. Using Poincaré duality, we find that

$$
H_{n-1}\left(L_{1} \backslash D_{1}\right) \approx H^{1}\left(L_{1} \backslash D_{1}, \partial D_{1}\right)
$$

and the latter vanishes since $L_{1} \backslash D_{1}$ is simply connected. We also have

$$
H_{n}\left(L_{1} \backslash D_{1}\right) \approx H^{0}\left(L_{1} \backslash D_{1}, \partial D_{1}\right)=0 .
$$

Therefore, the groups $H_{n-1}$ and $H_{n}$ are also zero, so $L_{1} \backslash D_{1}$ has the $\mathbb{Z} / 2$ homology of the $n$-disk.

Using Mayer-Vietoris we find that $L_{1}$ is a $\mathbb{Z} / 2$-homology sphere.

(c) Denote by $H \subset H^{1}\left(T^{m} \times Q ; \mathbb{Z}\right)$ the subgroup $H^{1}\left(T^{m} \times\{\mathrm{pt}\} ; \mathbb{Z}\right)$. The group $H$ is isomorphic to $\mathbb{Z}^{m}$ and any cohomology class $u \in H \backslash\{0\}$ obviously contains a closed non-vanishing 1-form on $T^{m} \times Q$. It follows by Theorem 1.3 that

$$
H_{*}\left(L \times T^{l}, p^{*} u\right)=0
$$

for every $u \in H \backslash\{0\}$.

Recall that we may suppose (using Remark 3.4) that $p$ is an epimorphism, which implies that $p^{*}$ is a monomorphism. Consider $H^{1}(L) \subset H^{1}\left(L \times T^{l}\right)$. We show that

$$
H^{1}(L) \cap p^{*}(H) \neq\{0\} .
$$


If not, then the composition

$$
\mathbb{Z}^{m} \approx p^{*} H \hookrightarrow H^{1}\left(L \times T^{l}\right) \stackrel{\mathrm{pr}}{\rightarrow} H^{1}\left(T^{l}\right) \approx \mathbb{Z}^{l}
$$

is a monomorphism, which is impossible, since $l<m$. We infer that there exists a nonvanishing class $p^{*} u=v \in H^{1}(L) \subset H^{1}\left(L \times T^{l}\right)$ such that

$$
H_{*}\left(L \times T^{l}, v\right)=0 .
$$

By applying Proposition 2.6 we obtain then $H_{*}(L, v)=0$. Finally, we apply Proposition 2.3 to get the desired conclusions on $\pi_{1}(L)$ and finish the proof.

Acknowledgements. I thank Michele Audin for many valuable discussions on Floer homology. I thank Agnes Gadbled and Alexandru Oancea, who carefully read the manuscript and helped me to improve it by their remarks and corrections. Finally, I thank the anonymous referee for his useful remarks and suggestions.

\section{References}

[1] V. I. Arnol'd, First steps in symplectic topology. Russ. Math. Surv. 41 (6) (1986), 1-21. Zbl 0649.58010 MR 0890489

[2] M. Audin, Fibrés normaux d'immersions en dimension double, points doubles d'immersions lagrangiennes et plongements totalement réels. Comment. Math. Helv. 63 (1988), 593-623. Zbl 0666.57024 MR 0966952

[3] L. Buhovsky, Homology of Lagrangian submanifolds in cotangent bundles. Israel J. Math. 143 (2004), 181-187. Zbl 1090.53063 MR 2106982

[4] A. Floer, Symplectic fixed points and holomorphic spheres. Comm. Math. Phys. 120 (4) (1989), 575-611. Zbl 0755.58022 MR 0987770

[5] A. Floer, Morse theory for Lagrangian intersections. J. Differential Geom. 28 (3) (1988), 513-547. Zbl 0674.57027 MR 0965228

[6] A. Floer, The unregularized gradient flow of the symplectic action. Comm. Pure Appl. Math. 41 (6) (1988), 775-813. Zbl 0633.53058 MR 0948771

[7] A. Floer, Witten's complex and infinite dimensional Morse theory. J. Differential. Geom. 30 (1989), 207-221. Zbl 0678.58012 MR 1001276

[8] R. H. Fox, Free differential calculus I. Ann. of Math. 57 ( 1953) 547-560; II, ibid. 59 (1954) 196-210. Zbl 0142.22303 MR 0053938 MR 0062125

[9] K. Fukaya, P. Seidel, I. Smith, Exact Lagrangian submanifolds in simply connected cotangent bundles. Invent. Math. 172 (1) (2008), 1-27. Zbl 1140.53036 MR 2385665

[10] K. Fukaya, P. Seidel, I. Smith, The symplectic geometry of cotangent bundles from a categorical viewpoint. In Homological mirror symmetry. New developments and perspectives, Lecture Notes in Phys. 757, Springer-Verlag, Berlin 2009, 1-26. Zbl 05555431 
[11] H. Hofer, D. Salamon, Floer homology and Novikov rings. In The Floer memorial volume, Progr. Math. 133, Birkhäuser, Basel 1995, 483-524. Zbl 0842.58029 MR 1362838

[12] F. Lalonde, J.-C. Sikorav, Sous-variétés lagrangiennes et lagrangiennes exactes des fibrés cotangents. Comment. Math. Helv. 66 (1991), 18-33. Zbl 0759.53022 MR 1090163

[13] F. Latour, Existence de 1-formes fermées non-singulières dans une classe de cohomologie de de Rham. Inst. Hautes Études Sci. Publ. Math. 80 (1995), 135-194. Zbl 0837.58002 MR 1320607

[14] H.V. Le, K. Ono, Symplectic fixed points, the Calabi invariant and Novikov homology. Topology 34 (1) (1995), 155-176. Zbl 0822.58019 MR 1308493

[15] D. Nadler, Microlocal branes are constructible sheaves. arXiv:math/0612399v4

[16] S. P. Novikov, Multivalued functions and functionals. An analogue of the Morse theory. Soviet. Math. Dokl. 24 (2) (1981), 222-226. Zbl 0505.58011 MR 0630459

[17] Y. G. Oh, Floer cohomology of Lagrangian intersections and pseudoholomorphic disks. I. Commun. Pure Appl. Math. 46 (1993), 949-993. Zbl 0795.58019 MR 1223659

[18] M. Pozniak Floer homology, Novikov rings and clean intersections. In Northern California Symplectic Geometry Seminar, Amer. Math. Soc. Transl. Ser. (2) 196, Amer. Math. Soc., Providence, RI, 1999, 119-181. Zbl 0948.57025 MR 1736217

[19] A. Ritter, Novikov symplectic cohomology and exact Lagrangian embeddings. Geom. Topol. 13 (2) (2009), 943-978. Zbl 05530196 MR 2470967

[20] P. Seidel, Exact Lagrangian submanifolds in $T^{*} S^{n}$ and the graded Kronecker quiver. In Different faces of geometry, Int. Math. Ser. (N. Y.) 3, Kluwer/Plenum, New York 2004, 349-364. Zbl 1070.53049 MR 2103000

[21] J.-C. Sikorav, Homologie de Novikov associée à une classe de cohomologie réelle de degré un. Thèse, Orsay 1987.

[22] D. Tischler, On fibering certain foliated manifolds over $S^{1}$. Topology 9 (1970), 153-154. Zbl 0177.52103 MR 0256413

[23] C. Viterbo, Exact Lagrange submanifolds, periodic orbits and the cohomology of free loop spaces. J. Differential Geom. 47 (3) (1997), 420-468. Zbl 0946.37017 MR 1617648

[24] C. Viterbo, Functors and computations in Floer homology with applications. I. Geom. Funct. Anal. 9 (5) (1999), 985-1033. Zbl 0954.57015 MR 1726235

[25] C. Viterbo, Intersection de sous-variétés lagrangiennes, fonctionnelles d'action et indice des systemes hamiltoniens. Bull. Soc. Math. France 115 (3) (1987), 361-390. Zbl 0639.58018 MR 0926533

Received October 16, 2007

Mihai Damian, Université Louis Pasteur, IRMA, 7, rue René Descartes, 67084 Strasbourg, France

E-mail: damian@math.u-strasbg.fr 